\title{
The effect of metallicity on the Cepheid Period-Luminosity relation from a Baade-Wesselink analysis of Cepheids in the Galaxy and in the Small Magellanic Cloud ${ }^{\star}, \star \star$
}

\author{
J. Storm ${ }^{1}$, B. W. Carney ${ }^{2}$, W. P. Gieren ${ }^{3}$, P. Fouqué ${ }^{4,5}$, D. W. Latham ${ }^{6}$, and A. M. Fry ${ }^{2}$ \\ 1 Astrophysikalisches Institut Potsdam, An der Sternwarte 16, 14482 Potsdam, Germany \\ e-mail: jstorm@aip.de \\ 2 Univ. of North Carolina at Chapel Hill, Dept. of Physics and Astronomy, Phillips Hall, Chapel Hill, NC-27599-3255, USA \\ e-mail: bruce@physics.unc.edu, anne.fry@integraonline.com \\ ${ }^{3}$ Universidad de Concepción, Departamento de Física, Casilla 160-C, Concepción, Chile \\ e-mail: wgieren@coma.cfm.udec.cl \\ ${ }^{4}$ Observatoire de Paris, LESIA, 5, place Jules Janssen, 92195 Meudon Cedex, France \\ 5 European Southern Observatory, Casilla 19001, Santiago 19, Chile \\ e-mail: pfouque@eso.org \\ ${ }^{6}$ Harvard-Smithsonian Center for Astrophysics, 60 Garden Street, Cambridge, Massachusetts 02138, USA \\ e-mail: dlatham@cfa.harvard.edu
}

Received 5 August 2003 / Accepted 14 November 2003

\begin{abstract}
We have applied the near-IR Barnes-Evans realization of the Baade-Wesselink method as calibrated by Fouqué \& Gieren (1997) to five metal-poor Cepheids with periods between 13 and 17 days in the Small Magellanic Cloud as well as to a sample of 34 Galactic Cepheids to determine the effect of metallicity on the period-luminosity (P-L) relation. For ten of the Galactic Cepheids we present new accurate and well sampled radial-velocity curves. The Baade-Wesselink analysis provides accurate individual distances and luminosities for the Cepheids in the two samples, allowing us to constrain directly, in a purely differential way, the metallicity effect on the Cepheid P-L relation. For the Galactic Cepheids we provide a new set of P-L relations which have zero-points in excellent agreement with astrometric and interferometric determinations. These relations can be used directly for the determination of distances to solar-metallicity samples of Cepheids in distant galaxies, circumventing any corrections for metallicity effects on the zero-point and slope of the P-L relation. We find evidence for both such metallicity effects in our data. Comparing our two samples of Cepheids at a mean period of about 15 days, we find a weak effect of metallicity on the luminosity similar to that adopted by the HST Key Project on the Extragalactic Distance Scale. The effect is smaller for the $V$ band, where we find $\Delta M_{V} / \Delta[\mathrm{Fe} / \mathrm{H}]=-0.21 \pm 0.19$, and larger for the Wesenheit index W, where we find $\Delta M_{\mathrm{W}} / \Delta[\mathrm{Fe} / \mathrm{H}]=-0.29 \pm 0.19$. For the $I$ and $K$ bands we find $\Delta M_{I} / \Delta[\mathrm{Fe} / \mathrm{H}]=-0.23 \pm 0.19$ and $\Delta M_{K} / \Delta[\mathrm{Fe} / \mathrm{H}]=$ $-0.21 \pm 0.19$, respectively. The error estimates are $1 \sigma$ statistical errors. It seems now well established that metal-poor Cepheids with periods longer than about 10 days are intrinsically fainter in all these bands than their metal-rich counterparts of identical period. Correcting the LMC distance estimate of Fouqué et al. (2003) for this metallicity effect leads to a revised LMC distance modulus of $(m-M)_{0}=18.48 \pm 0.07$, which is also in excellent agreement with the value of $(m-M)_{0}=18.50 \pm 0.10$ adopted by the Key Project. From our SMC Cepheid distances we determine the SMC distance to be $18.88 \pm 0.13$ mag irrespective of metallicity.
\end{abstract}

Key words. stars: distances - stars: fundamental parameters - Cepheids - Magellanic Clouds - distance scale

\section{Introduction}

Send offprint requests to: J. Storm, e-mail: jstorm@aip.de

* Some of the observations reported here were obtained with the Multiple Mirror Telescope, operated jointly by the Smithsonian Institution and the University of Arizona.

$\star \star$ Tables A.2-A.11 are only available in electronic form at the CDS via anonymous ftp to

cdsarc.u-strasbg.fr $(130.79 .128 .5)$ or via

http://cdsweb.u-strasbg.fr/cgi-bin/qcat?J/A+A/415/531
Cepheids remain one of the most accurate and well understood standard candles providing distances to galaxies out to the Virgo cluster. The Hubble Space Telescope has been used extensively to determine distances to such distant galaxies through the application of the Cepheid period-luminosity (P-L) relation. In this way it has been possible to calibrate secondary distance indicators which in turn are applicable to 
cosmological distances, thus enabling an accurate determination of the Hubble constant. The most recent papers in these series are Saha et al. (2001), who calibrate the peak luminosity of type Ia supernovae and find a Hubble constant of $H_{0}=$ $58.7 \pm 6.3$ (internal) $\mathrm{km} \mathrm{s}^{-1} \mathrm{Mpc}^{-1}$ and Freedman et al. 2001, who present the final results of the Hubble Space Telescope Key Project on the Extragalactic Distance Scale, and find a value of $H_{0}=72 \pm 8 \mathrm{~km} \mathrm{~s}^{-1} \mathrm{Mpc}^{-1}$.

These results are based on a few qualified assumptions. The first assumption is that the distance modulus to the Large Magellanic Cloud (LMC) is $\mu_{0}=18.50 \pm 0.10$, and the second assumption is that the P-L relation is only weakly dependent on metallicity. Freedman et al. (2001) adopt the value $\gamma_{V I}=\delta \mu_{0} / \delta[\mathrm{Fe} / \mathrm{H}]=-0.2 \pm 0.2 \mathrm{mag} \mathrm{dex}^{-1}$ based on the investigation of Kennicutt et al. (1998).

The first assumption has been the subject of extensive studies, but there is still not a complete consensus about the distance to this Galaxy, which is one of our nearest neighbours. Recent distance estimates to the LMC still range, embarrasingly enough, from 18.1 to 18.8 , see e.g. Benedict et al. (2002).

The metallicity effect has not been investigated quite as thoroughly and from both the observational and theoretical points of view the results have not been very accurate. This means that there is even doubt about the sign of the possible effect, and it is also not entirely clear if the slope of the P-L relation (Tammann et al. 2003) is affected.

The Baade-Wesselink method and its variants provide the means to determine accurate distances to individual Cepheids in contrast to the P-L relation which, due to the finite width of the instability strip, only provides accurate determinations for an ensemble of Cepheids. Several calibrations of the BaadeWesselink method based on interferometric diameters measured for red giant stars have appeared in the literature over the last decade, see e.g. Fouqué \& Gieren (1997, FG97 hereinafter), Di Benedetto (1997), or Arellano Ferro \& Rosenzweig (2000), and they show great promise for delivering accurate distances only weakly dependent on stellar atmosphere computations (Gieren et al. 2000). These calibrations are currently being confirmed by direct interferometric measurements of nearby Cepheids (Nordgren et al. 2002).

In the present work we investigate the difference in absolute magnitude between Cepheids with different metallicity. We do this by comparing absolute magnitudes for five metal-poor Cepheids in the Small Magellanic Cloud $([\mathrm{Fe} / \mathrm{H}]=$ -0.7) with solar-metallicity Cepheids in the Milky Way. The SMC Cepheids are the most metal-poor Cepheids which we have access to and provide a lever-arm in metallicity which is twice as large as for Cepheids in the LMC. The Galactic Cepheids are similarly the most metal-rich Cepheids which are sufficiently close to allow detailed observations. In both cases we determine the absolute magnitudes by applying the Infrared Surface-Brightness (hereinafter ISB) method calibrated by FG97. This method is a near-IR calibration of the Barnes-Evans (Barnes \& Evans 1976) variant of the Baade-Wesselink method.

By applying the same method to both samples of stars we can determine, in a purely differential way, the effect of metallicity, independent of the exact luminosity zero-point of the method.

In Sect. 2 we present the available observational data for the SMC sample of five Cepheids as well as for the sample of Galactic Cepheids which we will use for the comparison. We also discuss the reddening and reddening law for the two samples which is crucial for the final conclusions. New radialvelocity curves for ten of the Galactic Cepheids can be found in the Appendix.

In Sect. 3 we present the FG97 $F_{V},(V-K)$-version of the Barnes-Evans surface brightness method which we use, and we argue that the method itself is very insensitive to reddening and metallicity effects.

In Sect. 4 we present our results as they emerge from our application of the ISB method to the two samples of stars. We determine new Galactic P-L relations in the BVIJHKW bands based on the ISB analysis of the Galactic sample of stars. We compare the luminosities for the SMC stars to these relations and find a weak metallicity effect. We proceed to apply this metallicity effect to the LMC Cepheids and find a new metallicity-corrected distance modulus for the LMC.

In Sect. 5 we discuss the results in context with other determinations and we show that both the metallicity effect and the metallicity-corrected LMC distance modulus are in excellent agreement with the values adopted by the Key Project (Freedman et al. 2001).

\section{Observational parameters}

\subsection{The SMC Cepheid photometry and radial velocities}

The sample of five SMC Cepheids and their BVRIJK photometry and radial-velocity curves have been presented in full in Storm et al. (2004). Here we emphasize that the five SMC Cepheids are fundamental-mode pulsators with periods of approximately 15 days. With a sample of five stars at similar period, we have a good constraint on the location of the ridge line of the P-L relation at this period.

The optical light curves are based on CCD photometry presented in that paper as well as data from Udalski et al. (1999b), and the near-IR light curves are based on data obtained with various near-IR imaging cameras at the Las Campanas, Cerro Tololo and La Silla observatories.

The radial-velocity curves are based on high-resolution echelle spectra obtained mainly at the Las Campanas Observatory but supplemented with data from both Cerro Tololo and La Silla.

The $(V-K)$ color curve which is used for the BaadeWesselink analysis has been derived for each Cepheid by performing a low order Fourier fit to the mostly sinusoidal and low amplitude $K$ band light curve and determining the $K$-magnitude from the fit at every $V$ band observation as described in Storm et al. (2004). This procedure was necessary as the optical and near-IR data were not obtained simultaneously. 


\subsection{The Milky Way Cepheid photometry and radial velocities}

The reference sample of fundamental-mode Galactic Cepheids was selected on the availability of accurate $V$ and $K$ light curves as well as accurate radial velocity curves. For the present purpose it is important that the data points have small uncertainties and also that the phase coverage is good in all three observables. The data for these stars were compiled from the literature and by using the McMaster Cepheid database. In addition we have acquired extensive radial-velocity data for a number of these stars. These data are presented in the Appendix. The original sources for the data for each star can be found in Table 1.

We have also compiled the $B$ and $I$ data from these sources and have transformed the $I$ data to the Cousins system using the transformations from the Johnson system given by Caldwell et al. (1985) where necessary. We have finally also included the intensity mean $I$ values collected by Groenewegen (1999) for S Nor, 1 Car, and RS Pup.

\subsection{The reddening towards the Galactic Cepheids}

Fernie (1990) has combined the available reddening estimates toward Galactic Cepheids from many different photometric systems in a systematic way. He has furthermore determined a transformation from the resulting reddening scale to the cluster reddening scale of Feast \& Walker (1987). The latter scale is based on cluster OB star reddenings. We adopt these transformed reddenings so we are sure that we are on the same system as used for the ZAMS fitting distances to the clusters and thus that we can compare our absolute $V$ magnitudes with those found from ZAMS fitting to the clusters.

We have adopted the optical reddening laws from Caldwell $\&$ Coulson (1987) and Dean et al. (1978) and the near-IR reddening laws from Laney \& Stobie (1993).

We define the coefficient $R_{x}$ in the band $x$ from the absorption in the band $A_{x}$ by $A_{x}=R_{x} \times E(B-V)$ or $R_{x}=A_{x} / E(B-V)=$ $R_{V} \times A_{x} / A_{V}$.

We thus adopt the following relations:

$$
\begin{aligned}
& R_{V}=3.07+0.28(B-V)_{0}+0.04 E(B-V) \\
& R_{I}=1.82+0.205(B-V)_{0}+0.0225 E(B-V) \\
& R_{J}=0.249 \times R_{V} \\
& R_{H}=0.147 \times R_{V} \\
& R_{K}=0.091 \times R_{V} .
\end{aligned}
$$

For the reddening insensitive Wesenheit function $W_{V I}$ (Freedman et al. 2001) we have $W=V-R_{W}(V-I)$ where $R_{W}=1 /\left(1-\left(R_{I} / R_{V}\right)\right)$.

Computing the mean over the 32 Galactic calibrators we find that the dispersion in $R_{x}$ is very small $(0.036$ in $V)$ and we simply adopt constant values for $R_{x}$. We find $R_{V}=3.30$, $R_{I}=1.99, R_{W}=2.51, R_{J}=0.82, R_{H}=0.48$, and $R_{K}=0.30$.

We note that following Cardelli et al. (1989) $R_{W}$ would

\begin{tabular}{|c|c|c|c|}
\hline 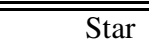 & $\overline{\bar{V}}$ & $\overline{K K}$ & " Radial velocity \\
\hline$\eta \mathrm{Aql}$ & 3,14 & 3,20 & $6,10,12$ \\
\hline WZ Car & 5,17 & 11,19 & 5 \\
\hline U Car & 5,17 & 11,20 & 5,12 \\
\hline $1 \mathrm{Car}$ & 17 & 11 & 12 \\
\hline VY Car & $5,13,17$ & 11,20 & 5,12 \\
\hline SU Cas & 3,14 & 3 & 2,6 \\
\hline KN Cen & 5 & 11 & 5 \\
\hline XX Cen & 5 & 11 & 12 \\
\hline VW Cen & 5,17 & 11 & 5 \\
\hline V Cen & 9,17 & 11,20 & 8,12 \\
\hline$\delta$ Cep & 3,14 & 3 & 6,10 \\
\hline X Cyg & 10,14 & 3,20 & 2,6 \\
\hline T Mon & 14 & 11 & 2,6 \\
\hline CV Mon & 14,17 & 11,20 & 6,16 \\
\hline UU Mus & 5,13 & 11 & 5 \\
\hline U Nor & $5,13,17$ & 11 & 5,18 \\
\hline S Nor & 17 & 11 & 2 \\
\hline V340 Nor & 22,7 & 11 & 2 \\
\hline BF Oph & 9,14 & 11 & $1,8,12$ \\
\hline LS Pup & 5 & 11,19 & 5 \\
\hline BN Pup & $5,13,17$ & 11,19 & 5 \\
\hline VZ Pup & 5,17 & 11,19 & 5 \\
\hline AQ Pup & 5,14 & $11,19,21$ & 1,5 \\
\hline RS Pup & 17 & $11,20,21$ & 6 \\
\hline RY Sco & $5,13,14,17$ & 11,20 & $1,5,12$ \\
\hline EV Sct & 14,17 & 11 & $2,6,15$ \\
\hline BB Sgr & 9,14 & 11,20 & 8 \\
\hline WZ Sgr & 14 & 11,20 & 1,5 \\
\hline U Sgr & 9,14 & 11 & 2,6 \\
\hline $\mathrm{T}$ Vel & 17,23 & 11 & 8,12 \\
\hline SW Vel & $13,14,17$ & 11 & 5,18 \\
\hline RY Vel & 5 & 11,20 & 5 \\
\hline RZ Vel & 5,13 & 11 & 5,12 \\
\hline SV Vul & 14 & $3,11,20$ & 6 \\
\hline
\end{tabular}
equal 2.45 , but we prefer the above value as it is based directly on Cepheid measurements.
Table 1. The list of the references which provided the $V$ and $K$ band photometry and the radial velocities.

1: Barnes et al. (1987); 2: Bersier et al. (1994); 3: Barnes et al. (1997); 4: Berdnikov \& Turner (1995); 5: Coulson \& Caldwell (1985a); 6: This work; 7: Eggen (1983); 8: Gieren (1981a); 9: Gieren (1981b); 10: Kiss (1998); 11: Laney \& Stobie (1992); 12: Lloyd Evans (1980); 13: Madore (1975); 14: Moffett \& Barnes (1984); 15: Metzger et al. (1991); 16: Metzger et al. (1992); 17: Pel (1976); 18: Pont et al. (1994); 19: Schechter et al. (1992); 20: Welch et al. (1984); 21: Welch (1985); 22: Coulson \& Caldwell (1985b) 23: Gieren (1985).

\subsection{The adopted metallicity}

It is well known that the Galactic Cepheids exhibit a range of metallicity values (see e.g. Fry \& Carney 1997 and Luck et al. 2003), and it is expected that the SMC Cepheids similarly span a range of values. However, as the SMC stars are faint from a high-resolution spectroscopic point of view, we do not have individual spectroscopic abundances for these stars yet. Instead we adopt an average value for our sample of stars as we are 
here mainly interested in the relative differences between the Milky Way Cepheids and the SMC Cepheids.

Luck et al. (1998) determined metallicities for six SMC Cepheids based on high-resolution spectroscopy. Combined with similar data for an additional 19 Cepheids from the literature these authors find the average value $[\mathrm{Fe} / \mathrm{H}]=-0.68$ with a standard deviation of $\sigma=0.13$. The same authors find from a similar study of Galactic Cepheids an average value of $[\mathrm{Fe} / \mathrm{H}]=-0.03$ based on their own sample of 11 stars, and an average value of $[\mathrm{Fe} / \mathrm{H}]=+0.03(\sigma=0.14)$ when averaged over an additional 58 stars from the literature. Combining these two numbers gives a metallicity offset of $\Delta[\mathrm{Fe} / \mathrm{H}]=$ $[\mathrm{Fe} / \mathrm{H}](\mathrm{SMC})-[\mathrm{Fe} / \mathrm{H}](\mathrm{MW})=-0.70$ which we will adopt in the following. It is clear that the intrinsic spread introduces an additional uncertainty on the final result, and individual metallicities for the stars would be preferable. The spread for the Galactic Cepheids should average out as the Baade-Wesselink analysis has been extended to a large number of stars, but for the five SMC stars we estimate an additional uncertainty of the mean of $\sigma=0.13 / \sqrt{5-1}=0.07 \mathrm{dex}$ for the adopted mean metallicity.

\subsection{The reddening towards the SMC Cepheids}

Bessell (1991) discusses in detail a large body of reddening investigations for the SMC and finds that the foreground reddening towards the SMC is smooth with a mean value of about $E(B-V)=0.05$. He argues that the average reddening within the SMC is of a similar size but notes that different methods and populations give different answers.

Zaritsky et al. (2002) find a mean absorption of $A_{V}=$ $0.18 \mathrm{mag}\left(E(B-V)=0.055\right.$ for $\left.R_{V}=3.30\right)$ for their sample of cool stars $\left(5500 \mathrm{~K} \leq T_{\text {eff }} \leq 6500 \mathrm{~K}\right)$ with very little spatial variation, but they also find a significantly higher mean absorption $\left(A_{V}=0.46\right)$ for their sample of hot stars.

Larsen et al. (2000) determined reddenings towards SMC B-stars using Strömgren photometry. They found foreground reddenings of $E(B-V)=0.07 \pm 0.02$ and mean reddenings of $E(B-V)=0.13$ for the two fields they investigated, in good agreement with the hot population results from Zaritsky et al. (2002) but larger than the foreground value of $E(B-V)=0.037$ given by Schlegel et al. (1998). The latter result is based on DIRBE measurements of foreground dust emission around the SMC. Larsen et al. (2000) also found that the reddening within each field exhibits significant scatter even on very small spatial scales (few arcsec) suggesting that reddening internal to the SMC and depth effects contribute significantly to the reddening of the individual stars.

Caldwell \& Coulson (1985) determined $E(B-V)=0.07$ for HV 1335 using $B V I$ reddenings based on the method of Dean et al. (1978) recalibrated for the lower metallicity of the SMC. Laney \& Stobie (1994) have adopted this value together with a value of $E(B-V)=0.06$ towards the other four stars in our sample based on the mean reddening calibrated with the Caldwell \& Coulson (1985) relation. These values have been tabulated in Table 2. In this table we have also tabulated the reddening values assigned by OGLE-2 (Udalski et al. 1999b) to the stars in common. These reddenings are based on red giant clump star colours and averaged on a field by field basis and they are only slightly larger than the values from Laney \& Stobie (1994).

The apparent complication of determining accurate reddenings towards SMC objects using a reddening-map-type approach shows that direct reddening determinations for individual objects are necessary for obtaining accurate luminosities, especially in the reddening-sensitive bands. As a consequence we have derived direct measures for the program stars using the observed mean $B V I$ data from Storm et al. (2004) and a recalibrated version of the Dean et al. (1978) method.

The Dean et al. (1978) method builds up an intrinsic (nonreddened) locus in a $(B-V)$ vs. $(V-I)$ diagram based on $B V I$ photometry of individual Cepheids. For each star the different phase points fall very narrowly along a straight line in the $(B-V),(V-I)$ plane. The straight lines for the different stars are shifted along the reddening vector to coincide and build up the intrinsic locus. The zero point is based on Cepheids in clusters with independent reddening estimates, typically based on B-stars. The locus of the intrinsic relation is sensitive to metallicity, and we have corrected this effect using model atmosphere computations. For this purpose Daniel Cordier has kindly evolved an 8 solar-mass model through the second and third crossing of the blue loop for three metallicities, $Z=$ 0.020 (solar), $Z=0.008$ (LMC), and $Z=0.004$ (SMC) using his model atmospheres and the BaSeL library (Lejeune et al. 1998). The paths of the second and third crossings are similar in the color-color diagram and can be well represented by a straight line. For each metallicity we have fitted a linear $(B-V)$ vs. $(V-I)$ relation, and we find a constant slope with metallicity but a blue shift in the intercept of -0.054 (LMC) and $-0.096(\mathrm{SMC})$ for fixed $(V-I)$, which corresponds to a shift in $E(B-V)$ of -0.08 and -0.14 for the LMC and SMC, respectively. These results are in excellent agreement with the original Dean et al. (1978) calibration. The main assumption underlying the method is that unreddened Cepheids indeed follow a common relation in the $(B-V),(V-I)$ plane independent of other parameters, luminosity (and thus period) in particular.

Due to the low absolute value of the reddening the exact choice of the reddening law does not cause a significant difference in the dereddened magnitudes. We assume that the Galactic and SMC reddening laws are quite similar and simply adopt the previously discussed Galactic law for the SMC Cepheids.

Applying these relations to the observed $B V I$ data we find reddening values ranging from -0.02 to 0.09 as tabulated in Table 2. The negative reddening for HV 1328 is clearly unphysical and a value of 0.0 has been adopted for this star in the following. The mean value for the remaining stars is 0.055 , in good agreement with the Laney \& Stobie (1994) values, but several of the values are quite low compared to the available foreground reddening estimates.

Assuming an uncertainty of $0.01 \mathrm{mag}$ for both the $(B-V)$ and $(V-I)$ colors gives an estimated uncertainty of about 0.02 mag on the individual estimates to which a similar amount of systematic uncertainty on the color zero points has to be added. A small global offset may still be present, but the 
Table 2. Reddening values for the stars from different authors.

\begin{tabular}{cccc}
\hline \hline ID & $\begin{array}{c}E(B-V) \\
\text { LS94 } \\
\text { mag }\end{array}$ & $\begin{array}{c}\text { OGLE-2 } \\
\text { mag }\end{array}$ & $\begin{array}{c}B V I^{3} \\
\text { mag }\end{array}$ \\
\hline HV 822 & 0.06 & 0.078 & +0.03 \\
HV 1328 & 0.06 & - & $-0.02^{4}$ \\
HV 1333 & 0.06 & - & +0.07 \\
HV 1335 & 0.07 & 0.070 & +0.09 \\
HV 1345 & 0.06 & 0.078 & +0.03 \\
\hline
\end{tabular}

1: Laney \& Stobie (1994); 2: Udalski et al. (1999b); 3: Using the $B V I$ calibration described in the text; 4 : This value is unphysical and a value of zero has been adopted.

reddening differences should be real, assuming that all our stars share the same metallicity.

For the reddening-sensitive bands the use of the individual reddenings results in a slightly smaller scatter for the derived $\mathrm{P}-\mathrm{L}$ relations.

We conclude that individual reddening determinations are still rather uncertain, but as there appears to be a significant intrinsic scatter in the SMC reddenings, we prefer these values over the average values available. We note that the zero-point might be slightly too low, but that we do not have an obviously better estimate available. We estimate an uncertainty on the $E(B-V)$ zero point of $0.03 \mathrm{mag}$.

\section{The Baade-Wesselink analysis}

\subsection{The infrared surface brightness-color relation}

Fouqué \& Gieren (1997) have recalibrated the Barnes-Evans surface-brightness relation (Barnes \& Evans 1976) using interferometrically determined radii for giants and supergiants. They have also extended the calibration to use not only the $(V-R)$ but also the $(V-K)$ and $(J-K)$ color indices. Combining the stellar angular diameters determined from the surface-brightness variation with the radius variation measured from radial-velocity curves they determine stellar radii and distances. In the following we will apply this method to our sample of galactic and SMC Cepheids.

Gieren et al. (1997) (hereinafter GFG97) applied the FG97 calibration to a sample of Cepheids belonging to Galactic open clusters. In this way they could compare the distance estimates from the FG97 method with independent estimates based on ZAMS fitting. They found excellent agreement (5\% in the distance) for the $F_{V},(V-K)$ and $F_{K},(J-K)$ calibrations whereas the distances based on the $F_{V},(V-R)$ calibration showed significant systematic and random errors. This supports the finding of Welch (1994) who argued strongly in favour of using near-IR calibrations of the surface brightness.

New interferometers have recently started to produce direct measurements of Cepheid mean radii (Kervella et al. 2001), and Cepheid radius variations (Lane et al. 2002). Nordgren et al. (2002) present 59 interferometric measurements for three nearby Cepheids and find a surface brightness-color calibration which is very similar (to within $4 \%$ in distance) to that of FG97. The direct interferometric observations of Cepheids show great promise for placing the calibration on an even firmer basis in the near future as the measurements are done directly on pulsating stars of the same color as the stars to be studied. For now we choose to use the FG97 calibration (Eq. (6)) as it is based on many more stars and on individual angular diameters which exhibit less scatter.

\subsection{The effect of metallicity and gravity}

The FG97 calibration is based on Galactic stars which all have about solar abundance but span a large range of surface gravities. A certain dependence on metallicity is to be expected as the surface brightness, which is a temperature measure, is calibrated as a function of a color index.

To investigate the significance of such an effect we have taken the FG97 calibration (Eq. (6)) and rearranged it to include the effective temperature (Eq. (8)).

$$
\begin{aligned}
F_{V} & =3.947-0.131(V-K)_{0} \\
& =4.2207-0.1 \times S_{V} \\
& =4.2207-0.1\left(42.207-10 \log T_{\mathrm{eff}}-B . C \cdot V\right)
\end{aligned}
$$

Using the Kurucz model atmospheres (ATLAS9, Kurucz 1993) we have determined a grid of colors, temperatures, and bolometric corrections for an assumed value of $\log g=1.5$ and for a range of different metallicities. For each metallicity we can then compute $F_{V}$ for a range of temperatures, and as we have corresponding values of $(V-K)_{0}$ we can re-derive the linear relation between $F_{V}$ and $(V-K)_{0}$ for each metallicity. The relation does not reproduce the empirical relation above exactly but gives instead $F_{V}=3.949( \pm 0.005)-0.126( \pm 0.002)(V-K)_{0}$ for an assumed solar metallicity. For an assumed metallicity of $[\mathrm{Fe} / \mathrm{H}]=-0.7$ the relation becomes $F_{V}=3.947-$ $0.125(V-K)_{0}$, which means that there is no significant difference between the two relations.

We have repeated the procedure for $\log g=0.75$ and $\log g=$ 2.25 thus covering a range in $\log g$ similar to that spanned by the Cepheids in question according to the relation $\log g=$ $2.620-1.142 \log P$ from Fernie (1995). Again the differences are small. For the star X Cyg we find a distance estimate which is larger by $0.03 \mathrm{mag}$ for the $\log g=0.75$ relation and smaller by 0.03 mag for the $\log g=2.25$ relation. This effect would tend to make the slope of the P-L relation slightly steeper, but the effect is only about half of the estimated uncertainty on the slopes derived in Sect. 4.2. We thus confirm the FG97 finding that stars with very different surface gravities follow the same $F_{V},(V-K)$ relation.

We conclude that at the present level of accuracy we can consider the ISB method to be insensitive to both metallicity and gravity effects. In the following we will therefore simply apply the same $F_{V}-(V-K)_{0}$ relation to both Galactic and SMC Cepheids and for all periods.

\subsection{The adopted p-factor}

The projection factor, $p$, for converting the radial velocities into pulsational velocities affects the final distances directly as it scales the radius variation. This means that an overestimate 
of the $p$-factor by $1 \%$ leads to an overestimate of the distance by $1 \%$ (0.02 mag in the distance modulus). Consequently, reliable results can only be obtained when the $p$-factor is well determined.

In reality the $p$-factor is not a simple geometric factor but does also depend on the structure of the stellar atmosphere, and the way the radial velocity is measured. Parsons (1972) computed the $p$-factor on the basis of line profiles derived from atmosphere models to take properly into account the effect of limb darkening, and the fact that the spectral lines are each formed over a range of optical depths in the atmosphere. He found values between 1.30 and 1.34 for Cepheids depending on the resolution of the spectrograph. Hindsley \& Bell (1986) investigated the effect of using a Griffin-type photoelectric radialvelocity spectrometer like CORAVEL (Baranne 1979) and they found a somewhat higher value, as well as a weak dependence on stellar surface temperature.

Gieren et al. (1993) adopted a simple linear weak dependence on temperature via the period,

$p=1.39-0.03 \log P$

based on the discussion of Hindsley \& Bell (1986, 1989), which we will also adopt here.

More recently Sabbey et al. (1995) have performed a careful observational and theoretical study of the line profiles, taking into account dynamic and non-LTE effects. They find that $p$ is not entirely constant with phase, and that the effect can amount to $7 \%$ (0.15 mag) in the distances determined with the method. Unfortunately, additional high-resolution and high-signal-to-noise spectra are needed to determine the phase behaviour of $p$, and for the SMC stars this is not possible. For lack of data and for consistency between the Galactic and SMC samples we use a $p$ which is constant for a given star, in agreement with LTE predictions. A constant $p$-factor is also supported by the results of GFG97; for their best-observed stars, like the 6-day-period star U Sgr, the agreement in shape between the linear displacement and angular diameter curves is excellent, which should not be the case if the $p$-factor was significantly phase-dependent. We note that in Sect. 4 we find that the match between photometric and spectroscopic angular diameters breaks down systematically around minimum radius, exactly where Sabbey et al. (1995) predicts the biggest deviations from an average $p$ value. In that section we argue that this phase interval should be disregarded, and this will minimize the possible effect on the derived distances.

A large fraction of the radial-velocity data for the Galactic stars were obtained using instruments of the photoelectric radial-velocity spectrometer type for which the previous relation is appropriate. Another significant fraction, in particular the data for the SMC stars from Storm et al. (2004) and the Galactic Cepheids presented in the Appendix using the CfA spectrographs, are based on high-resolution spectrometers where the radial velocity is determined by a software crosscorrelation using a template in $\log \lambda$ space.

We have the unique opportunity to determine directly the ratio between the $p$-factor for CORAVEL and the CfA spectrographs as the stars X Cyg and U Sgr have been observed extensively with both systems. Both stars have large velocity

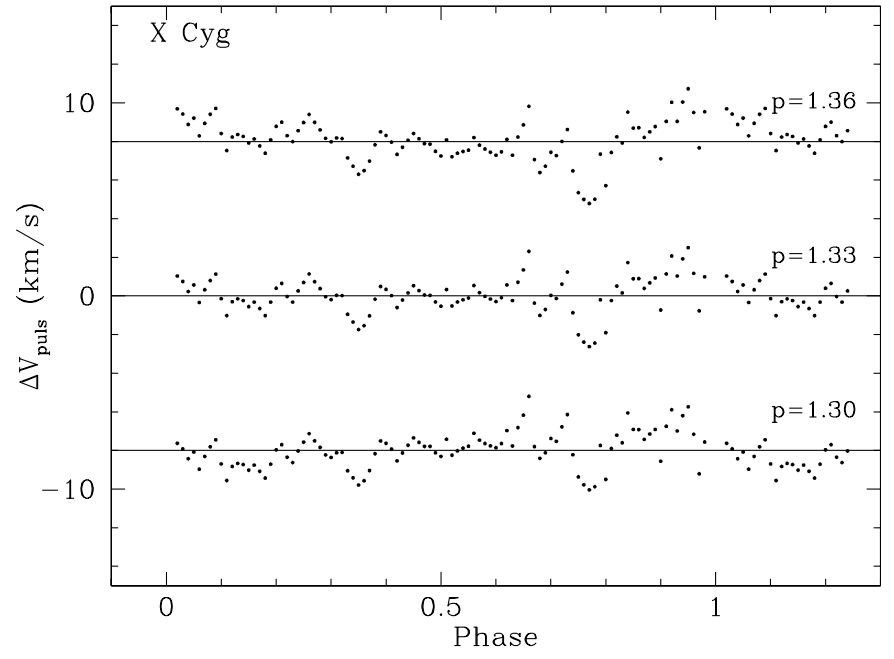

Fig. 1. The difference in pulsational velocity between the CfA and CORAVEL data sets for X Cyg using different $p$-factors for the CfA data and adopting a $p$-factor of 1.36 for the CORAVEL data. The zeropoints are arbitrary to separate the three examples.

amplitudes making them excellent calibration targets. Each of the data sets is reasonably well sampled over phase, so we have simply interpolated linearly in each of the datasets to generate for each star a pair of radial-velocity curves with a phase spacing of 0.01. To minimize any possible effect from amplitude variations for X Cyg we have only considered the data obtained in the period between HJD 2446000 and HJD 2447400. We have used the CORAVEL data as the reference and for the CfA velocities we have, for a number of different $p$ values, determined the differential pulsation velocity between the two spectrometers, $\Delta V_{\text {rad }, p}=V_{\text {rad }, p}(\mathrm{CfA})-V_{\text {rad }, p}(\mathrm{CORAVEL})$. For X Cyg we have plotted $\Delta V_{\text {rad, } p}$ against phase in Fig. 1. Assuming that $p$ is constant with phase, $\Delta V_{\text {rad, } p}$ should be constant as a function of phase for the appropriate ratio of $\eta=p_{\text {CfA }} / p_{\text {CORAVEL }}$ where $p_{\text {CORAVEL }}$ is computed from Eq. (9). We compute the slope of $\Delta V_{\mathrm{rad}, p}$ versus phase for the phase interval between minimum radial velocity $(\phi=0.05)$ and maximum velocity $(\phi=0.72)$ for a range of different $\eta$ values and determine the $\eta$ value where the slope is zero to be $\eta=1.34 / 1.354=0.990 \pm 0.02$. The result is robust and insensitive to the adopted phase interval. From Fig. 1 it is obvious that this is a difficult measurement and that relying just on a few points around maximum and minimum velocity will not lead to a significant result. For the short-period Cepheid U Sgr we similarly find $\eta=1.43 / 1.365=1.047 \pm 0.025$, where the larger error reflects the fact that the amplitude is smaller and the signal correspondingly less well constrained.

The difference in $\eta$ for the two stars is hardly significant and the average value of the two estimates is $\eta=1.019 \pm 0.02$, which is very close to unity. It is thus appropriate to use Eq. (9) also for these data.

For the SMC data set (Storm et al. 2004) the situation is very similar to that for the CfA data. Again the radial velocities have been derived from high-resolution $(0.02 \mathrm{~nm})$ spectra with a high dispersion $\left(0.2 \mathrm{~nm} \mathrm{~mm}^{-1}\right)$ and a sky exposure was used as the cross-correlation template. We have rerun the above 
test for the CfA data using a similar sky template, and again we found values very close to those for the CORAVEL data. We thus choose to adopt Eq. (9) for the SMC measurements as well.

\subsection{The fitting procedure}

There have been extensive discussions in the past (e.g. Laney $\&$ Stobie 1995, GFG97) regarding the proper fitting procedure for the radius variation versus angular-diameter fit. This has been an issue largely because the scatter in these diagrams, especially for optical colors, was very significant. In the near-IR the situation is in general much better as the scatter is significantly smaller and the choice of algorithm becomes much less critical. Since we are interested in determining the best relation between $\Delta R$ and $\theta$ and not in predicting $\theta$ from $\Delta R$ or the opposite, we follow the recommendation by Isobe et al. (1990) and use the bi-sector fit. Such a fit is shown in Fig. 2a. Had we chosen to use a linear least-squares fit with $\theta$ as the dependent variable as done by GFG97, we would have obtained distance moduli which are greater by approximately $0.02 \mathrm{mag}$.

When we perform the fit we also plot the resulting angular diameter as a function of phase, based on the resulting distance estimate (Fig. 2b). We notice that for many stars the fit in the phase region between 0.8 and 1.0 is very poor, and in almost all cases the photometric angular diameters show a larger radius than does the angular diameter curve derived from the radial-velocity measurements. In addition we see in many cases a significant bump in this phase interval. Clearly the method has a problem in this phase region. Sabbey et al. (1995) and others have argued that the $p$-factor could be significantly different especially in this phase region. Bersier et al. (1997) argue that non-LTE effects and increased microturbulence change the atmosphere structure and this could make the simple colorsurface brightness relation inadequate for this phase region. As we do not have a simple physical representation of the situation we prefer to disregard the phase region from 0.8 to 1.0 for all the stars. This does not affect our absolute calibrations of the Cepheid P-L relations significantly, but the scatter in the $\mathrm{P}-\mathrm{L}$ relations becomes slightly smaller.

For some stars we observe a slight phase shift between the angular diameter curves derived from photometry and spectroscopy as already noticed by GFG97. This shift results in a poorly defined linear relation between $\Delta R$ and $\theta$ which resembles a loop. The shifts, $\Delta \phi$, have been determined by minimizing the scatter of the fits and the values are tabulated in Table 3. It can be seen that the shifts are in general small and for most of the stars it is not even measurable. We do not have a physical explanation for the effect, but we do not believe that it is caused by a real phase difference between the photometric and spectroscopic data.

The application of the phase shifts marginally reduces the scatter around the final P-L relation. The effect on the slope of the P-L relation is small, but of the same size as the internal fitting error. It works in the direction of making the slopes in Table 4 shallower by about 0.15 than what would otherwise be predicted. Even though it is unsatisfactory that we do not
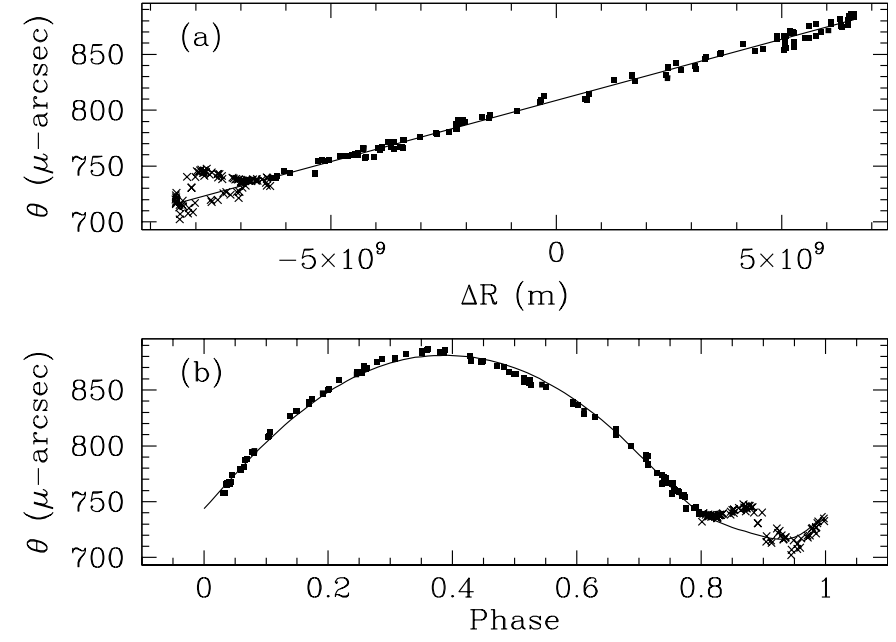

Fig. 2. The points represent the photometrically determined angular diameters for X Cyg and the line in panel a) shows the bi-sector fit to the filled points. The curve in panel $\mathbf{b}$ ) delineates the angular diameter obtained from integrating the radial velocity curve at the derived distance. Crosses represent points which were eliminated from the fit.

fully understand the effect, it is reassuring that the effects on the luminosities are limited and that only a few stars are affected.

\section{Results}

We have applied the procedure described in the previous section to both the sample of Galactic Cepheids and SMC Cepheids. The resulting radii, absolute magnitudes, and distances have been tabulated in Table 3 together with the adopted reddenings. The quoted errors in the table are $1 \sigma$ statistical errors.

\subsection{The luminosity zero-point}

For the Cepheids which are considered members of open clusters or associations we can compare the Cepheid distances which we have derived using the infrared surface brightness method with the distances found by ZAMS fitting. This is equivalent to comparing the luminosity zero-points of the two methods. Turner \& Burke (2002) have published the latest and most extensive list of ZAMS based distances to cluster and association Cepheids including a total of 46 stars.

Eighteen of these stars are also present in our sample and can be used to compare the two methods directly. U Car and SV Vul are outside the period interval which we have adopted for computing our P-L relations, so they have been eliminated, as has AQ Pup which deviates by $0.79 \pm 0.11 \mathrm{mag}$ and which is considered an uncertain cluster member. Based on the remaining fifteen stars we find a weighted mean distance difference of

$(m-M)_{\mathrm{ISB}}-(m-M)_{\mathrm{ZAMS}}=+0.01 \pm 0.06$

with a dispersion of $\sigma=0.23$. If we remove three additional outliers $(\delta$ Cep, $0.37 \pm 0.11$; BB Sgr, $0.49 \pm 0.08$; and U Car, $-0.45 \pm 0.05)$ for which the case for cluster membership is also 
Table 3. Physical parameters resulting from the near-IR surface brightness method applied to the samples of Galactic and SMC Cepheids.

\begin{tabular}{|c|c|c|c|c|c|c|c|c|c|c|c|c|c|c|}
\hline ID & $\log P$ & $\begin{array}{c}(m-M)_{0} \\
\text { mag }\end{array}$ & $\begin{array}{c}\sigma_{(m-M)} \\
\text { mag }\end{array}$ & $\begin{array}{r}R \\
R_{\odot}\end{array}$ & $\begin{array}{l}\sigma_{R} \\
R_{\odot}\end{array}$ & $\begin{array}{c}M_{B} \\
\text { mag }\end{array}$ & $\begin{array}{c}M_{V} \\
\text { mag }\end{array}$ & $\begin{array}{c}M_{I} \\
\text { mag }\end{array}$ & $\begin{array}{c}M_{J} \\
\text { mag }\end{array}$ & $\begin{array}{l}M_{H} \\
\mathrm{mag}\end{array}$ & $\begin{array}{c}M_{K} \\
\mathrm{mag}\end{array}$ & $\begin{array}{l}M_{W} \\
\text { mag }\end{array}$ & $\begin{array}{c}E(B-V) \\
\text { mag }\end{array}$ & $\overline{\Delta \phi}$ \\
\hline \multicolumn{15}{|c|}{ Milky Way } \\
\hline SU Cas & 0.289884 & 8.164 & 0.070 & 29.3 & 0.9 & -2.725 & -3.140 & -3.640 & -3.925 & -4.112 & -4.137 & -4.391 & 0.287 & 0.000 \\
\hline EV Sct & 0.490098 & 11.249 & 0.105 & 34.2 & 1.6 & -2.878 & -3.345 & -3.977 & -4.230 & -4.418 & -4.442 & -4.923 & 0.679 & 0.045 \\
\hline BF Oph & 0.609329 & 9.271 & 0.034 & 32.0 & 0.5 & -2.134 & -2.750 & -3.398 & -3.844 & -4.113 & -4.181 & -4.372 & 0.247 & 0.035 \\
\hline $\mathrm{T}$ Vel & 0.666501 & 9.802 & 0.060 & 33.6 & 0.9 & -2.048 & -2.692 & -3.370 & -3.887 & -4.179 & -4.260 & -4.392 & 0.281 & 0.000 \\
\hline$\delta$ Cep & 0.729678 & 7.085 & 0.044 & 42.0 & 0.9 & -2.870 & -3.431 & -4.060 & -4.471 & -4.752 & -4.808 & -5.007 & 0.092 & 0.000 \\
\hline CV Mon & 0.730685 & 10.988 & 0.034 & 40.3 & 0.6 & -2.460 & -3.038 & -3.797 & -4.263 & -4.547 & -4.647 & -4.932 & 0.714 & 0.015 \\
\hline V Cen & 0.739882 & 9.175 & 0.063 & 42.0 & 1.2 & -2.714 & -3.295 & -3.957 & -4.414 & -4.694 & -4.770 & -4.950 & 0.289 & 0.000 \\
\hline BB Sgr & 0.821971 & 9.519 & 0.028 & 49.8 & 0.6 & -2.817 & -3.518 & -4.262 & -4.724 & -5.029 & -5.102 & -5.382 & 0.284 & -0.035 \\
\hline U Sgr & 0.828997 & 8.837 & 0.021 & 47.7 & 0.5 & -2.785 & -3.477 & -4.213 & -4.668 & -4.950 & -5.023 & -5.317 & 0.403 & 0.000 \\
\hline$\eta \mathrm{Aql}$ & 0.855930 & 6.990 & 0.045 & 48.4 & 1.0 & -2.946 & -3.581 & -4.271 & -4.717 & -5.012 & -5.073 & -5.310 & 0.149 & 0.000 \\
\hline S Nor & 0.989194 & 9.908 & 0.032 & 70.7 & 1.0 & -3.345 & -4.101 & -4.856 & -5.411 & -5.734 & -5.818 & -5.995 & 0.189 & 0.000 \\
\hline XX Cen & 1.039 & 11.114 & 0.022 & 69.4 & 0.7 & -3.430 & -4.154 & -4.897 & -5.415 & -5.719 & -5.802 & -6.015 & 0.260 & -0.040 \\
\hline V340 Nor & 1.052579 & 11.145 & 0.185 & 67.1 & 5.7 & -2.985 & -3.814 & -4.679 & -5.225 & -5.577 & -5.670 & -5.980 & 0.315 & 0.000 \\
\hline UU Mus & 1.065819 & 12.590 & 0.084 & 74.1 & 2.9 & -3.426 & -4.159 & -4.926 & -5.495 & -5.813 & -5.905 & -6.079 & 0.413 & -0.005 \\
\hline U Nor & 1.101875 & 10.716 & 0.060 & 76.3 & 2.1 & -3.713 & -4.415 & -5.141 & -5.645 & -5.928 & -6.017 & -6.225 & 0.892 & 0.000 \\
\hline BN Pup & 1.135867 & 12.951 & 0.050 & 83.3 & 1.9 & -3.765 & -4.513 & -5.270 & -5.780 & -6.100 & -6.182 & -6.408 & 0.438 & 0.000 \\
\hline LS Pup & 1.150646 & 13.554 & 0.056 & 90.1 & 2.3 & -3.926 & -4.685 & -5.433 & -5.953 & -6.281 & -6.357 & -6.554 & 0.478 & 0.000 \\
\hline VW Cen & 1.177138 & 12.804 & 0.039 & 86.7 & 1.6 & -3.147 & -4.037 & -4.932 & -5.630 & -6.022 & -6.136 & -6.275 & 0.448 & 0.000 \\
\hline X Cyg & 1.214482 & 10.420 & 0.018 & 105.6 & 0.9 & -4.123 & -4.991 & -5.768 & -6.273 & -6.615 & -6.691 & -6.939 & 0.288 & 0.000 \\
\hline VY Car & 1.276818 & 11.499 & 0.022 & 112.8 & 1.1 & -3.929 & -4.846 & -5.702 & -6.324 & -6.679 & -6.783 & -6.994 & 0.243 & -0.020 \\
\hline RY Sco & 27 & 10.514 & & 99.9 & 1.6 & -4.394 & -5.060 & -5.806 & -6.269 & -6.540 & -6.623 & -6 . & .777 & 0.000 \\
\hline RZ Vel & 1.309564 & 11.021 & 0.029 & 114.8 & 1.5 & -4.250 & -5.042 & -5.826 & -6.408 & -6.735 & -6.827 & -7.000 & 0.335 & 0.000 \\
\hline WZ Sgr & 1.339443 & 11.287 & 0.047 & 121.8 & 2.6 & -3.874 & -4.801 & -5.721 & -6.381 & -6.765 & -6.881 & -7.103 & 0.467 & 0.000 \\
\hline WZ Car & 1.361977 & 12.918 & 0.066 & 112.1 & 3.4 & -4.142 & -4.918 & -5.718 & -6.322 & -6.661 & -6.745 & -6.922 & 0.384 & 0.000 \\
\hline VZ Pup & 1.364945 & 13.080 & 0.056 & 96.9 & 2.5 & -4.321 & -5.009 & -5.721 & -6.189 & -6.491 & -6.553 & -6.781 & 0.471 & 0.000 \\
\hline SW Vel & 1.370016 & 11.995 & 0.025 & 117.3 & 1.4 & -4.211 & -5.019 & -5.844 & -6.444 & -6.786 & -6.890 & -7.084 & 0.349 & -0.020 \\
\hline T Mon & 1.431915 & 10.815 & 0.055 & 149.5 & 3.8 & -4.403 & -5.372 & -6.247 & -6.892 & -7.274 & -7.375 & -7.564 & 0.209 & 0.000 \\
\hline RY Vel & 1.449158 & 12.019 & 0.032 & 139.9 & 2.1 & -4.693 & -5.501 & -6.302 & -6.885 & -7.183 & -7.277 & -7.506 & 0.562 & -0.005 \\
\hline AQ Pup & 1.478624 & 12.522 & 0.045 & 147.9 & 3.1 & -4.649 & -5.513 & -6.407 & -6.949 & -7.301 & -7.403 & -7.745 & 0.512 & -0.055 \\
\hline KN Cen & 1.531857 & 13.124 & 0.045 & 185.8 & 3.9 & -5.642 & -6.328 & -6.975 & -7.506 & -7.836 & -7.936 & -7.937 & 0.926 & 0.005 \\
\hline $1 \mathrm{Car}$ & 1.550855 & 8.990 & 0.032 & 201.8 & 2.9 & -4.712 & -5.821 & -6.772 & -7.454 & -7.871 & -7.965 & -8.205 & 0.170 & -0.040 \\
\hline U Car & 1.589083 & 10.973 & 0.032 & 161.6 & 2.4 & -4.725 & -5.617 & -6.480 & -7.104 & -7.452 & -7.559 & -7.779 & 0.283 & -0.050 \\
\hline RS Pup & 1.617420 & 11.561 & 0.064 & 208.0 & 6.1 & -5.047 & -6.015 & -6.962 & -7.599 & -7.967 & -8.084 & -8.384 & 0.446 & 0.000 \\
\hline SV Vul & 1.653162 & 12.102 & 0.037 & 224.0 & 3.8 & -5.876 & $\begin{array}{c}-6.752 \\
\text { SMC }\end{array}$ & -7.567 & -8.001 & -8.314 & -8.373 & -8.785 & 0.570 & -0.045 \\
\hline HV 1345 & 1.129638 & 18.828 & 0.081 & 78.4 & 2.9 & -3.502 & -4.166 & -4.926 & -5.515 & & -5.998 & -6.072 & 0.030 & 0.000 \\
\hline HV 1335 & 1.157807 & 18.875 & 0.082 & 72.6 & 2.8 & -3.808 & -4.365 & -5.045 & -5.545 & & -5.933 & -6.070 & 0.090 & 0.000 \\
\hline HV 1328 & 1.199645 & 18.732 & 0.087 & 80.4 & 3.2 & -4.051 & -4.617 & -5.295 & -5.747 & & -6.164 & -6.319 & 0.000 & 0.000 \\
\hline HV 1333 & 1.212014 & 19.388 & 0.080 & 100.3 & 3.7 & -4.271 & -4.913 & -5.660 & -6.171 & & -6.601 & -6.787 & 0.070 & 0.000 \\
\hline HV 822 & 1.223810 & 19.091 & 0.081 & 97.0 & 3.6 & -3.934 & -4.673 & -5.495 & -6.014 & & -6.473 & -6.729 & 0.030 & 0.000 \\
\hline
\end{tabular}

not considered very strong the dispersion decreases to $\sigma=0.12$ and the mean value changes marginally to $+0.05 \pm 0.04$.

Feast (1999) points out that the Hipparcos results indicate that the ZAMS fitting method is probably less well understood than previously assumed and that the zero-point can be uncertain by perhaps $0.2 \mathrm{mag}$. On the other hand we can conclude that the two methods are in excellent agreement and we take this as further support for the correctness of the luminosity zero-point for the ISB method and thereby for the zero-point of the resulting P-L relations.

Fouqué et al. (2003) have compared the latest Hipparcos determination of the zero-point of the P-L relations with the results from the surface brightness analysis of a sample of Galactic Cepheids. They find generally good agreement, lending additional support to the calibration of Fouqué \& Gieren (1997) and the zero-point of the resulting P-L relations. 
Table 4. The period-luminosity relations for the Galactic sample in different bands and the RMS around the fit.

\begin{tabular}{rcccc}
\hline \hline Band & $\alpha$ & $\beta$ & $\gamma$ & rms \\
\hline$B$ & $-2.74 \pm 0.12$ & $-3.30 \pm 0.03$ & $-3.79 \pm 0.03$ & 0.18 \\
$V$ & $-3.08 \pm 0.11$ & $-4.03 \pm 0.04$ & $-4.59 \pm 0.03$ & 0.17 \\
$I$ & $-3.30 \pm 0.10$ & $-4.79 \pm 0.03$ & $-5.38 \pm 0.03$ & 0.16 \\
$J$ & $-3.53 \pm 0.10$ & $-5.30 \pm 0.03$ & $-5.93 \pm 0.03$ & 0.15 \\
$H$ & $-3.63 \pm 0.11$ & $-5.61 \pm 0.03$ & $-6.26 \pm 0.03$ & 0.16 \\
$K$ & $-3.67 \pm 0.12$ & $-5.69 \pm 0.03$ & $-6.35 \pm 0.03$ & 0.15 \\
$W$ & $-3.63 \pm 0.11$ & $-5.92 \pm 0.04$ & $-6.58 \pm 0.03$ & 0.16 \\
\hline
\end{tabular}

\subsection{The $P$ - $L$ relations}

Based on the absolute magnitudes for the 32 Galactic Cepheids in the relevant period interval $(0.60<\log P<1.62)$ we rederive the $\mathrm{P}-\mathrm{L}$ relation in these bands in the form:

$M_{x}=\alpha(\log P-1.00)+\beta$

and

$M_{x}=\alpha(\log P-1.18)+\gamma$

where $M_{x}$ is the absolute magnitude in band $x$ and the offset in $\log P$ of 1.18 corresponds to the mean period of the SMC sample. The fit of the latter form provides a formal estimate of the uncertainty in the magnitude at the average period of our SMC sample. The fitted coefficients and their error estimates are tabulated in Table 4.

We have also determined the P-L relation for the Wesenheit function, W, as used by the HST Key Project (Freedman et al. 2001) and adopting our value of $R_{W}=2.51$ from Sect. 2.3. We have then defined $M_{W}=W_{V I}-(m-M)_{0}$ for our Galactic stars, where the distance modulus comes from our reddening corrected surface brightness analysis in Table 3, and proceeded as for the other bands.

The resulting relations provide a direct starting point for determining distances to the HST Key Project galaxies without any assumptions regarding the LMC distance and, as the metallicity difference between the Galaxy and the Key Project galaxies is, on average, smaller than between the LMC and the target galaxies, any metallicity effect will have a smaller effect on the derived distances. The relation based on the Wesenheit function allows us to derive $V$ - and $I$-based distances to the Key Project galaxies using the Wesenheit function to correct for reddening in the external galaxies. This approach requires a zero-point for our reddening scale for the Galactic Cepheids, whereas the Key Project (Freedman et al. 2001) can work purely differentially. We, however, have the advantage of being able to determine distances without assuming a distance to the LMC and with a smaller metallicity difference between the galaxies and the reference (the Galaxy).

We note that all the P-L relations which we have derived here are, as expected, in very good agreement with the relations derived by Gieren et al. (1998) which used the same method but a slightly smaller data set.

The absolute magnitudes versus $\log P$ and the fitted relations are shown in Figs. 3-5.

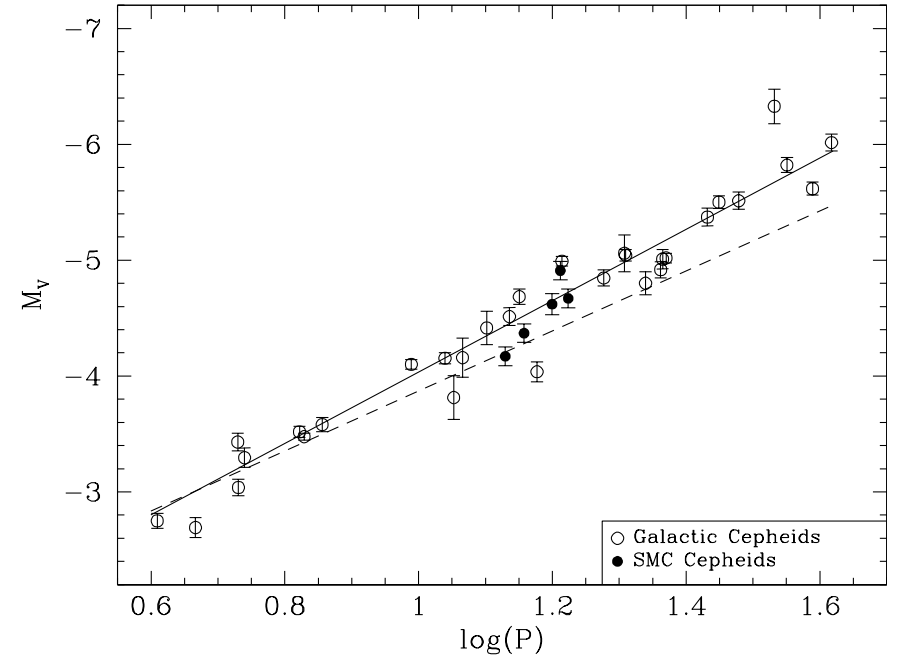

Fig. 3. The absolute $V$ magnitudes for the Galactic and SMC Cepheids as derived from the Baade-Wesselink analysis plotted against $\log P$. The dashed line represents the SMC relation based on OGLE data shifted to the adopted SMC distance (see Eq. (15)).

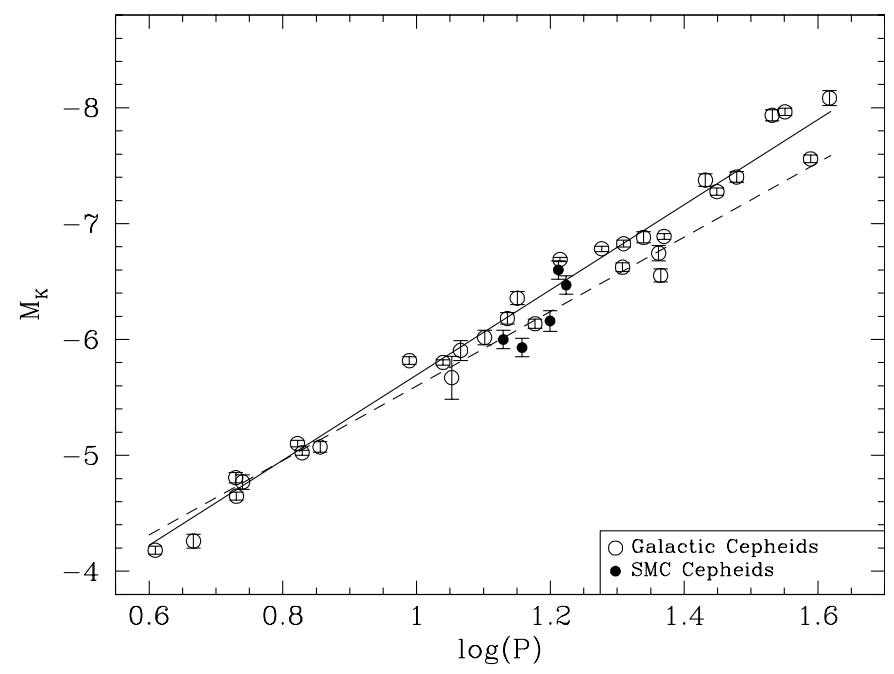

Fig. 4. The absolute $K$ magnitudes for the Galactic and SMC Cepheids as derived from the Baade-Wesselink analysis plotted against $\log P$. The dashed line represents the SMC relation based on Eq. (14) and shifted to the adopted SMC distance.

\subsection{The period-radius relation}

In Fig. 6 we have plotted the period-radius diagram from our data. The Galactic Cepheids define a linear relation

$\log R=0.77( \pm 0.02) \log P+1.05( \pm 0.03)$

which has been overplotted on the data points in the figure. This relation is, as expected, in excellent agreement with that of Gieren et al.(1998).

It can be seen that the SMC stars in our sample are on average slightly smaller than the corresponding Galactic stars. Three of the stars are very close to the Galactic locus and HV 1335 exhibits the largest deviation followed by HV 1328. HV 1328 however, is already expected to be an extreme case as it is very close to the blue edge of the instability strip and the 


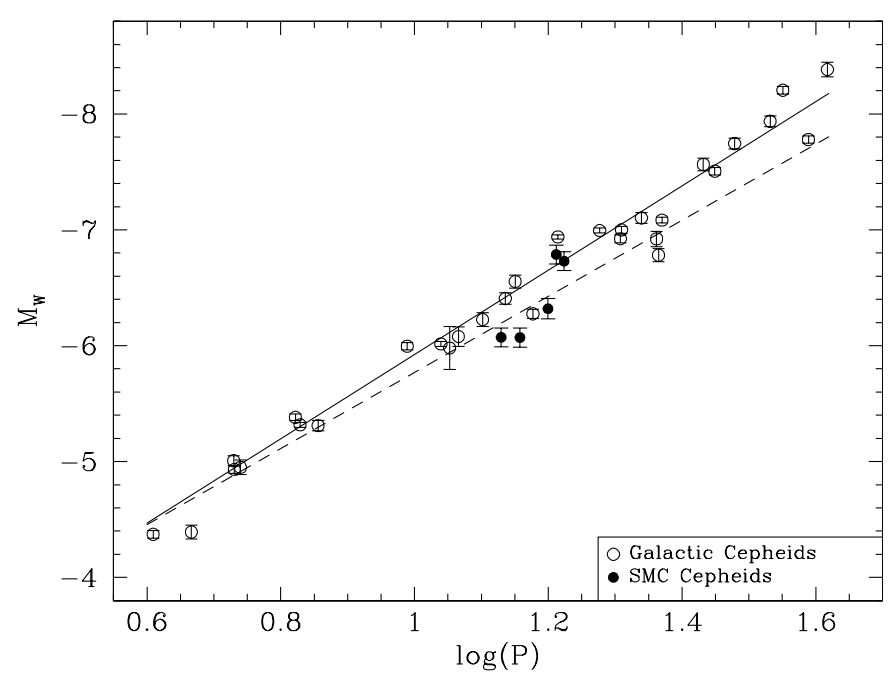

Fig. 5. The Wesenheit magnitudes for the Galactic and SMC Cepheids as derived from the Baade-Wesselink analysis plotted against $\log P$. The best linear fit has been overplotted. The dashed line represents the SMC relation based on OGLE data shifted to the adopted SMC distance (see Eq. (17)).

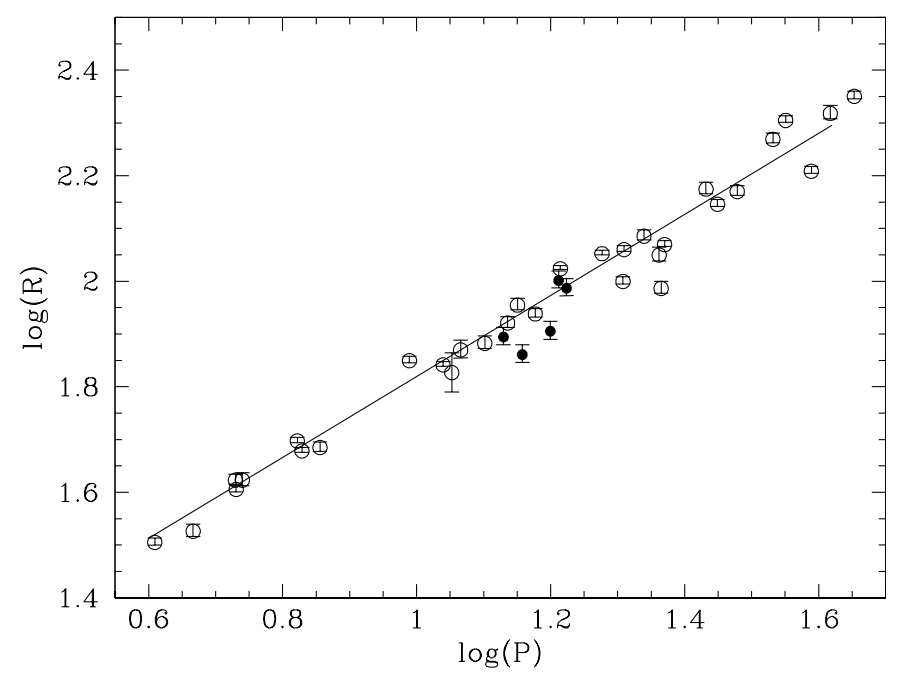

Fig. 6. The radii for the Galactic and SMC Cepheids as derived from the infrared surface brightness method plotted against $\log P$. The best fit straight line to the Galactic stars has been overplotted.

radial-velocity amplitude is extremely low $\left(A_{\mathrm{RV}}=23 \mathrm{~km} \mathrm{~s}^{-1}\right.$, Storm et al. 2004) for its period.

\subsection{The distance to the SMC}

The individual distance moduli to the present sample of SMC stars, as measured by the surface brightness method, are tabulated in Table 3, and the weighted mean distance modulus is $(m-M)_{0}=19.00 \pm 0.12$. This distance estimate is very robust to errors in the reddening estimate. Assuming a mean reddening of 0.00 or 0.12 would lead to moduli of 18.96 and 19.03 respectively.

To obtain the distance to the SMC itself, we formally have to take into consideration the depth effects in the SMC. Laney \& Stobie (1994) used the simple geometrical model of

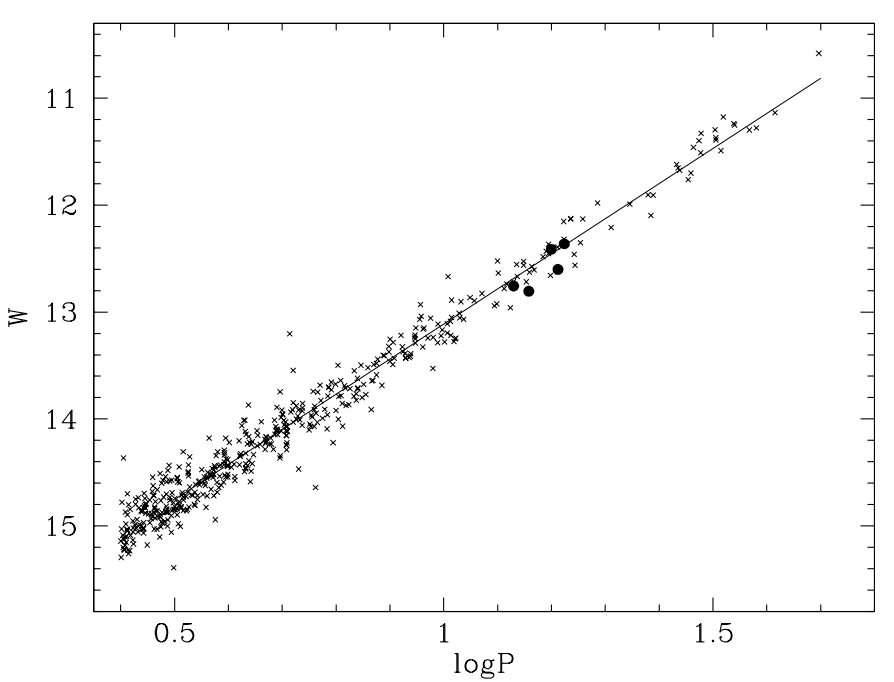

Fig. 7. The $W$-indices for the OGLE-2 sample plotted agains $\log P$. Our sample of stars has been overplotted as filled circles. The straight line shows the linear regression to the OGLE-2 data.

Caldwell \& Laney (1991) to derive corrections to the moduli of SMC Cepheids, including four of the stars in our sample. They found the values -0.142 for $\mathrm{HV} 1328,-0.126$ for HV 1333, -0.122 for HV 1335 , and -0.098 for HV 1345 . We have used the same method to derive a correction for the last star, HV 822, for which we find a value of -0.093 . We have also looked at the OGLE-2 $W-\log P$ relation and found an effect of similar size and direction as that determined by Caldwell \& Laney (1991). However, as the OGLE sample does not extend as far from the SMC center as the stars used by Caldwell \& Laney we adopt the latter values as they are based on a wider spatial baseline. The mean offset for our five stars is $-0.116 \pm 0.009$ mag in the sense that the distance to the bulk of the SMC is shorter by this amount than the distance to these five stars. In Fig. 7 the $W-\log P$ relation for the OGLE-2 sample is shown with our sample overplotted. The $W$-index for the OGLE sample has been computed using our value of $R_{W}=2.51$ for consistency. It is evident that our sample is slightly fainter than the ridge line of the OGLE-2 sample, by approximately $0.1 \mathrm{mag}$, further justifying the application of the depth correction.

Applying the individual offsets to the moduli in Table 3 and computing the weighted mean value gives an SMC distance modulus of $(m-M)_{0}=18.88 \pm 0.12 \mathrm{mag}$. Due to the limited sample size and the fact that the depth effect is a statistical effect of a size comparable to the spread around the ridge line, we add in quadrature an additional error of 0.07 mag to the resulting mean value leading to a best estimate of the SMC distance modulus of $(m-M)_{0}=18.88 \pm 0.14 \mathrm{mag}$.

\subsection{The slopes of the $P-L$ relations}

We can compare the slopes for the Galactic P-L relations which we have derived in Sect. 4.2 with the slopes derived for the SMC Cepheids on the basis of the dereddened data on fundamental-mode Cepheids in the OGLE-2 data base (Udalski et al. 1999b). Fouqué et al. (2003) found that the OGLE-2 reddenings for LMC Cepheids were too large when compared to 
Table 5. The period-luminosity relations for the 488 OGLE-2 SMC Cepheids in the period interval $0.4<\log P<1.7$ in different bands. A few outliers have been rejected before the fits. A reddening of $E(B-V)=E(B-V)_{\mathrm{OGLE}}-0.025$ has been assumed.

\begin{tabular}{cccc}
\hline \hline Band & $\alpha$ & $\beta$ & rms \\
\hline$V$ & $-2.590 \pm 0.047$ & $+15.010 \pm 0.019$ & 0.288 \\
$I$ & $-2.865 \pm 0.036$ & $+14.252 \pm 0.014$ & 0.220 \\
$W$ & $-3.283 \pm 0.023$ & $+13.112 \pm 0.009$ & 0.144 \\
\hline
\end{tabular}

other calibrations. We have included the OGLE-2 reddening estimates for the three stars in common with our sample in Table 2. The values are slightly larger than the Laney \& Stobie (1994) values and also larger than our BVI based estimates which we have adopted for our sample. To formally bring the two samples on the same reddening system we have simply offset the reddenings for the OGLE- 2 stars by -0.025 mag. We then extract the stars in the period interval $0.4<\log P<1.7$ and fit a P-L relation using linear regression after rejecting between one and five outlying points for each relation. The resulting fits are tabulated in Table 5. In the optical bands the scatter is rather large but in the $W$-index, which is insensitive to reddening, the rms around the fit decreases to 0.14 mag, even smaller than for our Galactic sample. This suggests that the increased width of the other P-L relations in the optical bands are not introduced by depth effects but rather by uncertainties in the individual reddening estimates.

In the $K$ band we can use the $K$ band P-L relation from Groenewegen (2000):

$K_{0}=-3.212 \pm 0.033 \log P+16.494 \pm 0.026$.

This relation is based on single or few phase measurements from the DENIS (Epchtein et al. 1999) and 2MASS (Jarrett et al. 2000) surveys.

Adopting our best estimate of the SMC distance of $(m-M)_{0}=18.88$ and combining this with the OGLE-2 relations from Table 5, and the $K$ band relation from Groenewegen (2000) we derive the relations

$$
\begin{aligned}
& M_{V}(\mathrm{SMC})=-2.590(\log P-1)-3.87 \\
& M_{I}(\mathrm{SMC})=-2.865(\log P-1)-4.63 \\
& M_{W}(\mathrm{SMC})=-3.283(\log P-1)-5.77 \\
& M_{K}(\mathrm{SMC})=-3.212(\log P-1)-5.60 .
\end{aligned}
$$

The dashed lines in Figs. 3-5 show these relations on top of the Galactic relations. It is obvious that the slopes of the SMC relations are less steep than the relations based on the Galactic sample. The difference is significant for all the photometric bands, and could be an effect of the difference in metallicity between the two samples. Evidence for such a significant dependence of the P-L slopes on metallicity has also recently been found by Tammann et al. (2003).

If the difference in slope is real and indeed dependent on metallicity, then it will have significant consequences for using the P-L relation as a distance estimator. In the relevant period range from $\log P=0.6$ to $\log P=1.6$ the effect in the
Table 6. The period-luminosity relations for the Galactic Cepheids but forcing the slope to be the one derived from the SMC sample.

\begin{tabular}{cccc}
\hline \hline Band & $\alpha$ & $\beta$ & rms \\
\hline$V$ & -2.590 & $-4.103 \pm 0.046$ & 0.22 \\
$I$ & -2.865 & $-4.854 \pm 0.043$ & 0.21 \\
$W$ & -3.283 & $-5.990 \pm 0.042$ & 0.20 \\
$K$ & -3.212 & $-5.775 \pm 0.044$ & 0.20 \\
\hline
\end{tabular}

$W$-index would amount to more than 0.3 mag as the slopes differ by $-3.283-(-3.63)=0.35$.

Udalski et al. (2001) find that the slopes are similar for the three low-metallicity galaxies IC $1613([\mathrm{Fe} / \mathrm{H}]=-1.0)$, SMC $([\mathrm{Fe} / \mathrm{H}]=-0.7)$, and $\mathrm{LMC}([\mathrm{Fe} / \mathrm{H}]=-0.3)$, and they argue that there is no metallicity effect on the P-L relations. However, they do not have a solar-metallicity galaxy in their sample so if we are really seeing a metallicity effect, then it might not be a simple linear effect in $[\mathrm{Fe} / \mathrm{H}]$.

It should be kept in mind that for periods shorter than about 10 days there is a significant risk for contaminating the sample of fundamental-mode pulsators with first-overtone pulsators which would be brighter at a given period. Udalski et al. (1999b) have used Fourier parameters to discriminate between pulsation modes, which works well for most periods, except in the interval $\log P=0.6$ to 0.8 . This could, in principle, change the observed slope. For the use for extra-galactic distances short-period Cepheids are currently not so interesting as they are too faint, so we can conveniently circumvent this possible problem by simply disregarding stars with periods shorter than 10 days $(\log P=1$.). Similarly, we make a cut at the long period end at $\log P=1.5$ to avoid possible problems with the most luminous stars which are not relevant for our 15-day-period stars. The slopes for the SMC and Galactic samples indeed become more similar, but the error estimates also become much larger due to the more limited baseline, so the significance of any difference in slopes becomes very small. The luminosity differences which we will derive in the next section change only by a few hundredth of magnitude, so we have decided to simply employ the full relations from above.

\subsection{The metallicity effect on Cepheid luminosities}

We can now investigate the metallicity effect on the zero-point of the P-L relation using three different approaches.

In the first approach we adopt the slopes of the SMC relations as the "true" slopes and fit only the zero-point for the Galactic sample. The resulting zero-points are tabulated in Table 6. For the optical bands we can compare these zeropoints with the zero-points based on the OGLE-2 sample for an assumed distance modulus of $(m-M)_{0}(\mathrm{SMC})=18.88$ as given in Eqs. (15)-(17). The difference $\Delta M=M_{\mathrm{MW}}-M_{\mathrm{SMC}}$ is tabulated in Col. 2 of Table 7. The effect is about $-0.22 \pm 0.14 \mathrm{mag}$ in both $V$ and $I$ with a contribution to the uncertainty of 0.13 from the distance estimate and 0.04 from the linear fit. In $W$ the effect is very similar, $-0.21 \pm 0.14$ and in the $K$ band we can make the comparison with the Groenewegen (2000) 
Table 7. The magnitude offset between the Galactic and SMC Cepheids for the different approaches mentioned in the text.

\begin{tabular}{lcccc}
\hline \hline \multicolumn{1}{c}{ Method } & $\begin{array}{c}\Delta M_{V} \\
\mathrm{mag}\end{array}$ & $\begin{array}{c}\Delta M_{I} \\
\mathrm{mag}\end{array}$ & $\begin{array}{c}\Delta M_{K} \\
\mathrm{mag}\end{array}$ & $\begin{array}{c}\Delta M_{W} \\
\mathrm{mag}\end{array}$ \\
\hline Fixed SMC slope, ZP fit only & -0.23 & -0.21 & -0.16 & -0.21 \\
Free fits, for $\log P=1.0$ & -0.16 & -0.16 & -0.09 & -0.16 \\
Free fits, for $\log P=1.18$ & -0.25 & -0.24 & -0.18 & -0.22 \\
Free fits, for $\log P=1.3$ & -0.31 & -0.29 & -0.23 & -0.26 \\
Direct comparison of sample & -0.06 & -0.10 & -0.13 & -0.19 \\
& & & & \\
Best estimate (see text) & -0.15 & -0.16 & -0.15 & -0.20 \\
\hline
\end{tabular}

relation from Eq. (18) where we find a very similar effect of $\Delta M_{K}=-0.16 \pm 0.14$.

In the second approach we use the free fits (slope and zeropoint) for the two samples and perform the comparison at different periods. At $\log P=1.18$, which is the average period of the SMC sample of Cepheids for which we have determined surface brightness distances, we find values of $\Delta M=-0.25$ for $V$ and $I$ with a similar uncertainty as above. Due to the different slopes of the two relations the effect is less at $\log P=1.0$ and larger at $\log P=1.3$. For completeness these values have been included in Table 7. As in the first approach we have compared the Galactic $K$-band relation with the Groenewegen (2000) relation and the result is again similar to the one obtained for the optical bands.

In the third approach we make a direct comparison between our Baade-Wesselink sample stars and the Galactic sample independently of the OGLE-2 sample. In this way we can investigate the metallicity effect in all the bands available, including the reddening-insensitive $K$ band, without having to make any assumptions regarding a possible difference in the slopes of the two P-L relations, and we also avoid the application of a correction for possible depth effects between the two samples. For each SMC star we simply compute the expected absolute magnitude from the Galactic relation. This value is then compared with the absolute magnitude returned by the BaadeWesselink analysis of the star. The offsets are again given in Table 7 where it can be seen that the effect in $V$ and $I$ is smaller than found from the previous two approaches by about $0.2 \mathrm{mag}$ in $V$ and $0.12 \mathrm{mag}$ in $I$ while the $K$ and $W$ offsets show very good agreement with the two previous methods. This result adds further support to the size and direction of the geometric depth correction which has been applied in Sect. 4.4.

Having light curves in the BVIJHK bands we can check the wavelength dependence of the magnitude offsets. In Table 8 we give the values for three different values for the assumed mean reddening. As is expected, the values in the table show that any conclusion regarding the wavelength dependence of the magnitude offsets is directly dependent on the assumed reddening. For an (unrealistic) reddening of $E(B-V)=0.0$ the offset is almost constant with wavelength, but as the reddening is increased the metallicity sensitivity becomes a steeper and steeper function of wavelength.
Table 8. The mean magnitude offsets from direct comparison of the five $\log P \approx 1.18$ SMC stars with the Galactic relations, for three different assumed values of the mean reddening.

\begin{tabular}{cccc}
\hline \hline$E(B-V)$ & 0.00 & 0.06 & 0.12 \\
Band & $\Delta M$ & $\Delta M$ & $\Delta M$ \\
& mag & mag & mag \\
\hline$B$ & -0.12 & +0.18 & +0.47 \\
$V$ & -0.24 & +0.00 & +0.24 \\
$I$ & -0.23 & -0.07 & +0.09 \\
$J$ & -0.21 & -0.13 & -0.04 \\
$K$ & -0.17 & -0.12 & -0.06 \\
$W$ & -0.22 & -0.18 & -0.14 \\
\hline
\end{tabular}

We note that the three methods described above all give the same offsets in the $K$ and $W$ indices, and that the $K$ and $W$ offsets agree well within the errors with each other. The $V$ and $I$ band results are more dependent on accurate reddening estimates but give basically the same offsets as the reddening-independent indices, at least for the first two methods. The third method suggests a smaller effect in $V$ and $I$, but this is hardly significant.

It should be stressed that we have direct measurements of the offsets only at periods near $\log P=1.18$. At this period the two first methods are almost equivalent and they can hardly be considered independent, which is also reflected in the excellent agreement between the two sets of values. Our best estimates for the offsets are thus computed as the weighted mean of the first two approaches, each with weight 0.5 , and the last approach with a weight of unity. These estimates are also given in Table 7.

Assuming a metallicity difference between the Milky Way sample and the SMC sample of $0.7 \pm 0.07$ dex as discussed in Sect. 2.4, and adopting the individual reddenings from Table 2, we have measured the effect for 15-day-period Cepheids to be $\Delta M_{V} / \Delta[\mathrm{Fe} / \mathrm{H}]=-0.21 \pm 0.19, \Delta M_{I} / \Delta[\mathrm{Fe} / \mathrm{H}]=-0.23 \pm 0.19$, $\Delta M_{W} / \Delta[\mathrm{Fe} / \mathrm{H}]=-0.29 \pm 0.19$, and $\Delta M_{K} / \Delta[\mathrm{Fe} / \mathrm{H}]=-0.21 \pm$ 0.12 where the minus sign indicates that the absolute magnitude of a more metal-poor star is fainter than a metal-rich counterpart of equal period. The error estimates are $1 \sigma$ statistical errors which have been propagated from the SMC distance error estimate and the metallicity error estimate. The metallicity effect is barely significant but in good agreement with the value adopted by Freedman et al. (2001).

\subsection{The distance to the $L M C$}

Fouqué et al. (2003) have derived P-L relations for the OGLE-2 stars in the LMC. They have forced the reddening to a common value of $E(B-V)=0.10$ to be in agreement with the Hubble key project. They did not apply any correction for metallicity but used the LMC slope of the P-L relations as the slope for the Galactic Cepheids and simply fitted the zero-point for the Galactic Cepheids on this basis. We follow the same procedure here and assume that the slopes can be considered equal, and apply our metallicity corrections to the 
Table 9. The distance modulus to the LMC in different bands before (Fouqué et al. 2003) and after correction for metallicity.

\begin{tabular}{rcc}
\hline \hline Band & $(m-M)_{0}$ & $(m-M)_{0, \mathrm{c}}$ \\
\hline$V$ & $18.536 \pm 0.048$ & $18.47 \pm 0.07$ \\
$I$ & $18.530 \pm 0.041$ & $18.45 \pm 0.07$ \\
$K$ & $18.567 \pm 0.048$ & $18.50 \pm 0.07$ \\
$W$ & $18.549 \pm 0.036$ & $18.46 \pm 0.07$ \\
\hline
\end{tabular}

Fouqué et al. (2003) distances. We assume that the metallicity effect is linear with $[\mathrm{Fe} / \mathrm{H}]$ and simply apply the metallicity effect for each index as determined in the previous section to the LMC moduli, under the assumption that $[\mathrm{Fe} / \mathrm{H}]_{\mathrm{LMC}}=-0.3$. The corrected distance moduli in the different bands can be found in Table 9 where our error estimate includes a contribution from the uncertainty in the metallicity correction of $0.3 \times 0.19=0.057$ added in quadrature .

The values agree well within the stated errors. The $W$-index already combines the $V$ and $I$ data in an optimum way, so to obtain a distance modulus free of reddening uncertainties we simply compute a weighted mean of the $W$ and $K$ results, which leads to a best estimate of the LMC distance modulus of $(m-M)_{0, c}=18.48 \pm 0.07$. The error estimate is a formal error which does not take into account possible systematic effects like differences in the slopes between the Galactic and LMC Cepheid P-L relations.

We note that the metallicity-corrected distance modulus is in good agreement with the ISB distance of $(m-M)_{0}=18.42 \pm$ 0.10 to the Cepheid HV 12198 in the LMC cluster NGC 1866 from Gieren et al. (2000) and is also in excellent agreement with the canonical value of $18.50 \pm 0.1$ adopted by Freedman et al. (2001).

\section{Discussion}

There have been several attempts to determine the metallicity effect on the Cepheid P-L relation empirically, but it has proven exceedingly difficult to reach truly conclusive results.

Freedman \& Madore (1990) observed three fields at different galactocentric distances in M 31, and thus at different metallicities. They found that the effect, if at all present, was not significant. This analysis and the results were later disputed by Gould (1994) who used a more sophisticated statistical treatment of the data and found a strong effect $(\Delta \mu / \Delta[\mathrm{Fe} / \mathrm{H}]=$ $\left.-0.88 \pm 0.16 \mathrm{mag} \mathrm{dex}^{-1}\right)$. Kennicutt et al. (1998) rediscussed these analyses pointing out that the assumed metallicity range was incorrect, but even then they had to conclude that this data set was not conclusive. Beaulieu et al. (1997) and Sasselov et al. (1997) applied a multi-variate analysis to the EROS sample of SMC and LMC Cepheids to determine an effect which is equivalent to $\Delta M_{W} / \Delta[\mathrm{Fe} / \mathrm{H}]=-0.44_{-0.2}^{+0.1} \mathrm{mag} \mathrm{dex}^{-1}$. Kochanek (1997) used a similar technique for a sample of 17 galaxies and Kennicutt et al. (1998) summarize the results as leading to $\gamma_{V I}=\delta \mu_{0} / \delta[\mathrm{O} / \mathrm{H}]=-0.4 \pm 0.2 \mathrm{mag} \mathrm{dex}^{-1}$. Kennicutt et al. (1998) used a technique similar to the differential approach of Freedman \& Madore (1990) in the Galaxy M 101 and found $\gamma_{V I}=-0.24 \pm 0.15 \mathrm{mag} \mathrm{dex}^{-1}$.
Udalski et al. (2001) found a zero effect when comparing the very low metallicity $([\mathrm{Fe} / \mathrm{H}]=-1.0)$ Galaxy IC 1613 with the LMC. This result depends critically on the assumed RR Lyrae luminosity-metallicity relation which is itself a topic of debate. As the Galaxies have such low metallicity, the result is also not necessarily representative of the solar-metallicity galaxies observed by the Key Project, especially if the effect turns out not to be a linear function of $[\mathrm{Fe} / \mathrm{H}]$. If the effect happens to be linear in $Z$, the heavy-element fraction, then an extrapolation from the LMC to the Galaxy would involve a factor of two as the Galactic Cepheids have a $Z$ twice as big as that of the LMC Cepheids.

Freedman et al. (2001) have summarized the empirical evidence and assumed that the slope of the P-L relation is universal, and finally adopted a value of $\gamma_{V I}=-0.2 \pm 0.2$ mag dex $^{-1}$.

In the light of our present results, and those discussed in Fouqué et al. (2003), we share the concern of Tammann et al. (2003) that the slope can be significantly different among populations. We have determined our offsets at a mean period of $\log P=1.18$ (15 days) which is not the same as the mean period of $\log P \approx 1.5$ of most Key Project Cepheid samples. If the slopes indeed do differ by as much as suggested by our previous analysis, then our offsets would be larger by approximately $0.1 \mathrm{mag}$ when applied to samples of $\operatorname{long}(\log P=1.5)$ period Cepheids.

When we compare our offset in the $W$-index with that from the Key Project, we find that our values are in excellent agreement well within the (admittedly large) errors. Including the slope variation and performing the comparison at $\log P=1.5$ would make the difference larger. In $W$ we would find an offset between the Galactic and SMC samples of 0.32 mag which would result in $\Delta W / \Delta[\mathrm{Fe} / \mathrm{H}]=-0.46 \pm 0.19$, which is very large, but still within the errors.

On the theoretical side extensive efforts over the last few years by several groups have not yet reached a consensus, and the situation is maybe even more confusing than from the empirical point of view.

Bono et al. (1999) have developed sequences of fullamplitude non-linear, convective models for a large range of parameters. These models have been further developed and analyzed in a number of papers. In the latest paper in the series, Fiorentino et al. (2002), it is argued that not only are Cepheid luminosities affected by metallicity, but the Helium content also plays a significant role. The metallicity effect is furthermore not a simple linear function in metallicity, and even the sign of the effect changes with abundance. These findings suggest that the problem might well be significantly more complicated than the simple linear relation which has so far been assumed for the empirical investigations. Unfortunately these computations suggest that metal-poor Cepheids are brighter than metal-rich Cepheids for the metallicities and periods considered in the present work.

An alternative theoretical approach using linear nonadiabatic models has been developed by Saio \& Gautschy (1998), by Alibert et al. (1999) and by Baraffe \& Alibert (2001). The first authors find a negligible metallicity effect on the P-L relation while the latter authors find a small effect of about $0.1 \mathrm{mag}$ for the $V, I, J$, and $K$ bands in the sense that 
metal-rich Cepheids are brighter than metal-poor ones. This is in good agreement with our results.

Sandage et al. (1999) find similarly, from a theoretical approach, that Cepheids with periods between 10 and 30 days show a mild metallicity effect. They estimate the effect to be $\Delta B / \Delta[\mathrm{Fe} / \mathrm{H}]=+0.03 \mathrm{mag} \mathrm{dex}^{-1}, \Delta V / \Delta[\mathrm{Fe} / \mathrm{H}]=$ $-0.08 \mathrm{mag} \mathrm{dex}^{-1}$, and $\Delta I / \Delta[\mathrm{Fe} / \mathrm{H}]=-0.10 \mathrm{mag} \mathrm{dex}^{-1}$ such that metal-rich stars are brighter in the $V$ and $I$ bands, similarly to what we find.

It is reassuring that most of the independent empirical methods lead to similar results, but the error bars are still too large to distinguish among the available theoretical models. The sign of the effect for Cepheids of period longer than about 10 days seems now to be reasonably well established, at least from an empirical point of view, especially for the reddening insensitive $K$ - and $W$-indices. In addition to the large empirical uncertainties it is also a serious concern that there is a distinct possibility of a difference in P-L slope between the populations. If such a difference is indeed present, the direct application of the Galactic P-L relation presented here to the metal-rich extragalactic Cepheid samples and the LMC P-L relation to the metal-poor samples, would allow this potential problem to be circumvented altogether as the metallicity difference between the samples would be small. Such an approach will depend on the adopted reddenings for the Galactic stars, but this effect will be small in the reddening-insensitive $W$ and $K$ P-L relations.

To place tighter constraints on the metallicity effect on both the zero point and the slope of the P-L relation it would be very worthwhile to extend this work to longer-period Cepheids (35-50 days) in the SMC. This would allow a direct comparison of the slopes of the P-L relations for the two different metallicity populations using the same method. It would also allow a comparison of the absolute magnitudes at a period which better resembles the mean period of the Key Project sample which becomes particularly important if a significant slope difference is indeed present.

\section{Summary}

We have presented Baade-Wesselink-based absolute magnitudes for a sample of 34 Galactic Cepheids, and derived $\mathrm{P}-\mathrm{L}$ relations spanning the period range $\log P=0.6$ to 1.62 . For samples of solar-metallicity Cepheids in other galaxies these relations allow a direct distance determination without the need for an assumed LMC distance and without the need for a significant correction for metallicity.

We find some evidence for a difference in slope of the P-L relations for the OGLE SMC sample and our Galactic Cepheid sample, suggesting that the metallicity effect might also affect the slopes of the P-L relations.

Using stellar atmosphere models we have investigated the metallicity effect on the infrared surface brightness method and we find only a negligible effect. On this basis we find, on a purely differential basis, that our sample of SMC Cepheids with periods around 15 days are intrinsically slightly fainter than Galactic Cepheids with similar periods.

Assuming that this effect is caused by the difference in metallicity between the two samples and assuming that this relation is linear over the range of metallicities in question we find for the reddening-insensitive indices that $\Delta M_{K} / \Delta[\mathrm{Fe} / \mathrm{H}]=$ $-0.21 \pm 0.19$ and $\Delta M_{W} / \Delta[\mathrm{Fe} / \mathrm{H}]=-0.29 \pm 0.19$. For our adopted reddening towards the SMC Cepheids we find a similar effect for the reddening-sensitive indices, namely $\Delta M_{V}=$ $-0.21 \pm 0.19$ and $\Delta M_{I}=-0.23 \pm 0.19$. We consider the adopted reddening a best estimate but realize that, if anything, the reddening could be stronger. This would cause the metallicity effect in the reddening-sensitive bands to be even closer to zero.

We have determined the distance to the SMC based on the ISB distances to individual Cepheids and by correcting for depth effects using a geometric model. We find $(m-M)_{0}=$ $18.88 \pm 0.13$.

Fouqué et al. (2003) have determined the distance to the LMC by applying the Galactic P-L relations from above to the OGLE data (Udalski et al. 1999a). We have corrected this distance estimate for the effect of metallicity and find a final best estimate of $(m-M)_{0}=18.48 \pm 0.07$ (internal error only).

We finally conclude that our results support the two fundamental assumptions of the Hubble Telescope Key Project on the Extragalactic Distance scale, namely that the LMC distance modulus is $18.50 \pm 0.10$ and that the metallicity effect in the $\mathrm{W}_{V I}$ index based distance is $\gamma_{V I}=-0.2 \pm 0.2 \mathrm{mag} \mathrm{dex}^{-1}$. However, if there is a significant metallicity effect on the slope of the P-L relation, we would find a stronger metallicity effect and a shorter LMC distance. In this case a direct application of the reddening-insensitive Galactic P-L relations to the extragalactic Cepheids would provide an attractive alternative to using the LMC as an intermediate stepping stone.

Acknowledgements. This research has made use of the SIMBAD database, operated at CDS, Strasbourg, France, NASA's Astrophysics Data System Bibliographic Services, and the McMaster Cepheid Photometry and Radial Velocity Data Archive. WPG acknowledges support for this work from the chilean FONDAP Center for Astrophysics 15010003. BWC thanks the U.S. National Science Foundation for grants AST-8920742 and AST-9800427 to the University of North Carolina for support for this research.

\section{Appendix A: New radial velocities}

New measurements of the radial velocities for ten of the Galactic cepheids have been obtained with the CfA Digital Speedometers Latham (1985, 1992). Three nearly identical echelle spectrographs have been used, on the Multiple Mirror Telescope and 1.5-m Tillinghast Reflector at the F. L. Whipple Observatory atop Mt. Hopkins, Arizona, and on the $1.5-\mathrm{m}$ Wyeth Reflector located at the Oak Ridge Observatory in the town of Harvard, Massachusetts. Photon-counting intensified Reticon detectors were used to record about $45 \AA$ of spectrum in a single order centered near $5187 \AA$. The spectral resolution is about $8.5 \mathrm{~km} \mathrm{~s}^{-1}$ for all the CfA spectra, and the signal-to-noise ratios range from about 5 to 50 per resolution element.

Radial velocities were extracted from the observed spectra using the one-dimensional correlation package r2rvsao 

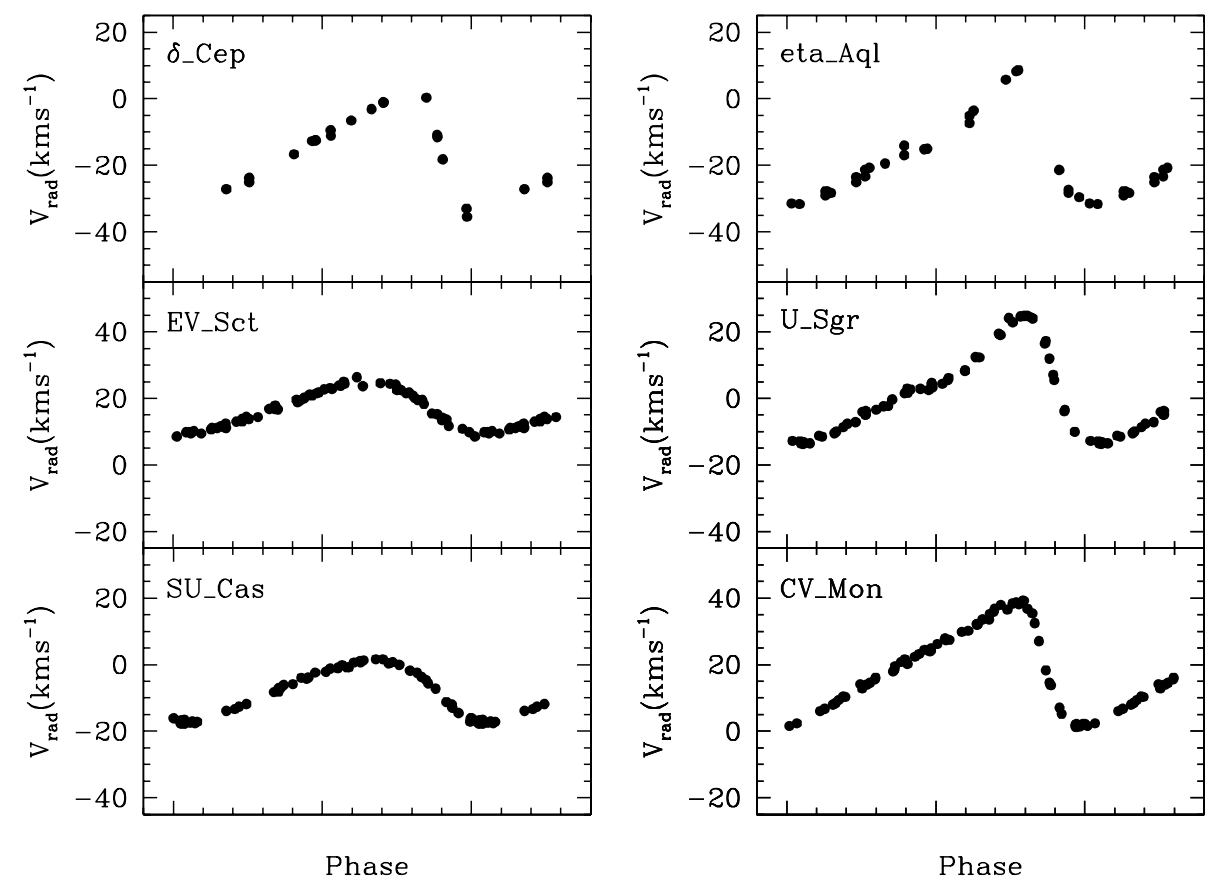

Fig. A.1. The radial velocity curves for the stars tabulated in Tables A.2-A.7.

Table A.1. The best-fitting template parameters, the number of observations, $N_{\text {obs }}$, and the time span of the observations. The last two columns give the rotational velocity and the systemic velocity.

\begin{tabular}{rcccccr}
\hline \hline $\begin{array}{r}\text { ID } \\
\text { K }\end{array}$ & $\begin{array}{c}T_{\text {eff }} \\
\mathrm{K}\end{array}$ & & & $\begin{array}{c}\text { log }(g) \\
\text { days }\end{array}$ & $\begin{array}{c}N_{\text {obs }} \\
\mathrm{km} \mathrm{s}^{-1}\end{array}$ & $\begin{array}{c}\gamma \\
\mathrm{km} \mathrm{s}^{-1}\end{array}$ \\
\hline SU Cas & 6500 & 1.5 & 53 & 944 & 15.0 & -7.3 \\
EV Sct & 6250 & 1.5 & 67 & 890 & 22.3 & 17.3 \\
$\delta$ Cep & 5750 & 1.5 & 20 & 126 & 15.2 & -16.3 \\
CV Mon & 6000 & 1.5 & 64 & 862 & 17.1 & 19.4 \\
U Sgr & 5750 & 1.5 & 57 & 749 & 19.7 & 2.8 \\
$\eta$ Aql & 5750 & 1.5 & 26 & 467 & 18.3 & -15.5 \\
X Cyg & 5250 & 0.5 & 75 & 806 & 21.2 & 7.5 \\
T Mon & 5250 & 0.5 & 88 & 937 & 21.9 & 20.3 \\
RS Pup & 5000 & 0.5 & 43 & 718 & 20.2 & 24.7 \\
SV Vul & 5250 & 0.5 & 75 & 839 & 22.4 & -1.3 \\
\hline
\end{tabular}

(Kurtz \& Mink 1998) running inside the IRAF $^{1}$ environment. For the templates we used a new grid of synthetic spectra (Morse \& Kurucz, priv. comm.) calculated using the latest Kurucz model atmospheres. The new grid of synthetic templates incorporates several improvements compared to the older grid that we used for several years (Nordström et al. 1994; Latham et al. 2002). To select the optimum synthetic template for each star we adopted solar metallicity and ran correlations for an appropriate range of effective temperature, $T_{\text {eff }}$, surface gravity, $\log g$, and rotational velocity. The $T_{\text {eff }}$ and $\log g$ for the templates that gave the highest average value for the

1 IRAF (Image Reduction and Analysis Facility) is distributed by the National Optical Astronomy Observatories, which are operated by the Association of Universities for Research in Astronomy, Inc., under contract with the National Science Foundation. peak correlation coefficient for the individual stars are listed in Table A.1. Of course, both $T_{\text {eff }}$ and $\log g$ vary systematically during the pulsational cycle of a Cepheid, so the values that we adopted for the template parameters can only represent some kind of average approximation. Similarly, the width of the observed correlation peaks vary significantly as a function of pulsational phase. One obvious source of variation in the observed line broadening with phase is the change in the pulsational velocity and its projection as one moves from the center to the edge of the disk. A more sophisticated approach to the analysis of cepheid velocities would be to match calculated spectra to each individual velocity and thereby derive $T_{\text {eff }}$ and $\log g$ at each observed phase in the pulsational cycle. One can even imagine an approach that would add an extra dimension to the grid of synthetic template spectra, namely the pulsational velocity, taking into account explicitly the effect of changing projection factor across the disk.

The template parameters derived from the CfA spectra follow the expected trend in the sense that the cepheids with longer periods are also cooler and have weaker surface gravity. Although it is reassuring that our procedure picks stellar parameters that appear to have some connection with reality, we must caution that our procedure was designed to optimize the radial-velocity determinations, not to determine the fundamental astrophysical characteristics of our stars. In particular, if the actual metallicity of a star is significantly different from solar, the $T_{\text {eff }}$ and $\log g$ values that give the best correlations will exhibit systematic errors. Furthermore, the most luminous cepheids in our sample give the best correlations for synthetic spectra at the edge of our grid at $\log g=0.5$.

The resulting radial-velocity data is tabulated for each star in Tables A.2-A.11 and the corresponding radial-velocity curves are plotted in Figs. A.1-A.2. Here the excellent coverage for most of the stars is apparent as well as the 

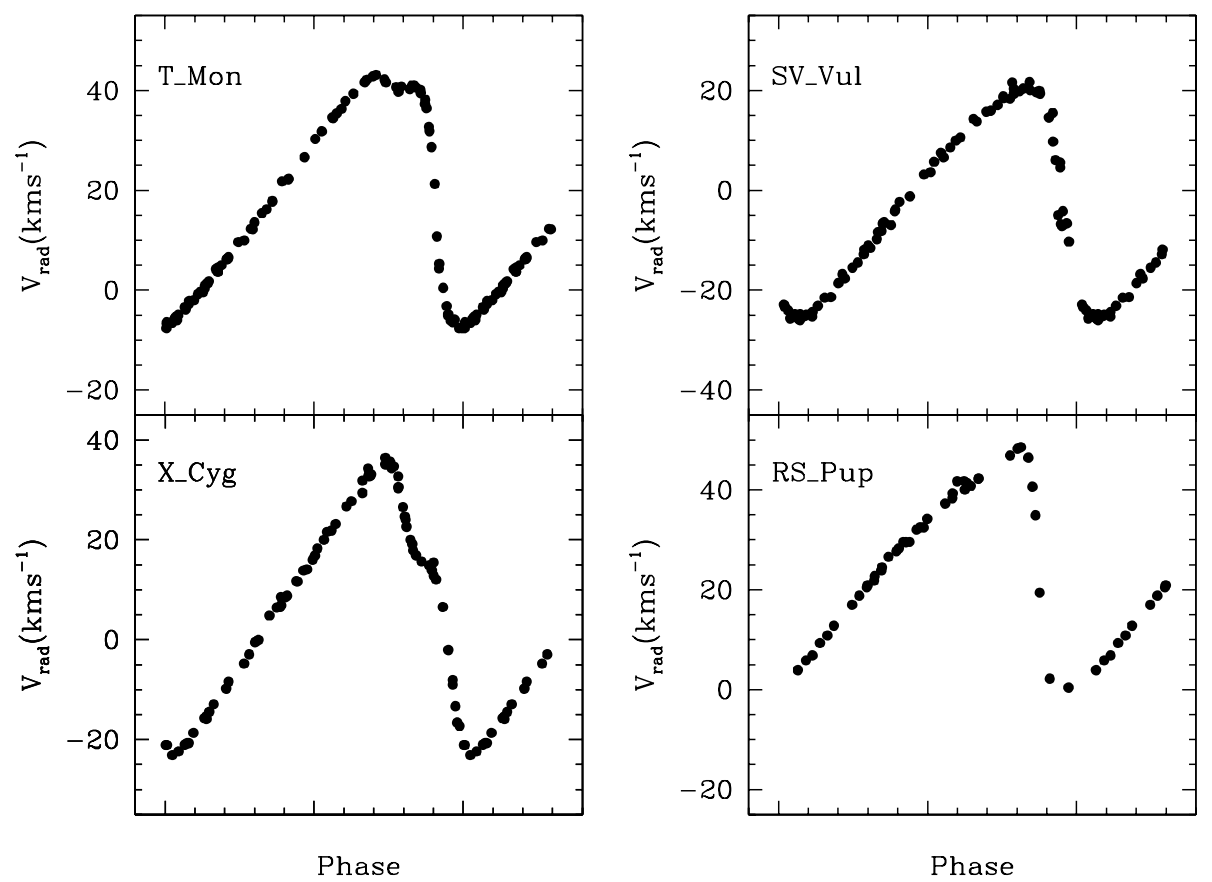

Fig. A.2. The radial velocity curves for the stars tabulated in Tables A.8-A.11.

low scatter. Note that the estimated errors are typically less than $0.5 \mathrm{~km} \mathrm{~s}^{-1}$.

\section{References}

Alibert, Y., Baraffe, I., Hauschildt, P., \& Allard, F. 1999, A\&A, 344, 551

Arellano Ferro, A., \& Rosenzweig, P. 2000, MNRAS, 315, 296

Baranne, A., Mayor, M., \& Poncet, J. L. 1979, Vistas Astr., 23, 279

Baraffe, I., \& Alibert, Y. 2001, A\&A, 371, 592

Barnes, T. G., \& Evans, D. S. 1976, MNRAS, 174, 489

Barnes, T. G., Moffett, T. J., \& Slovak, M. H. 1987, ApJS, 65, 307

Barnes, T. G., Fernley, J. A., Frueh, M. L., et al. 1997, PASP, 109, 645

Benedict, G. F., McArthur, B. E., Fredrick, L. W., et al. 2002, AJ, 123, 473

Berdnikov, L. N., \& Turner, D. G. 1995, Pis'ma Astr. Zh., 21, 803

Bersier, D., Burki, G., Mayor, M., \& Duquennoy, A. 1994, A\&AS, 108,25

Bersier, D., Burki, G., \& Kurucz, R. L. 1997, A\&A, 320, 228

Bessell, M. S. 1991, A\&A, 242, L17

Beaulieu, J. P., Sasselov, D. D., Renault, C., et al. 1997, A\&A, 318, L47

Bono, G., Marconi, M., \& Stellingwerf, R. F. 1999, ApJS, 122, 167

Caldwell, J. A. R., \& Coulson, I. M. 1985, MNRAS, 212, 879

Caldwell, J. A. R., Coulson, I. M., \& Gieren, W. P. 1985, ApJS, 57, 595

Cardelli, J. A., Clayton, G. C., \& Mathis, J. S. 1989, ApJ, 345, 245

Caldwell, J. A. R., \& Coulson, I. M. 1987, AJ, 93, 1090

Caldwell, J. A. R., \& Laney, C. D. 1991, in Proc. IAU Symp. 148, ed. R. Haynes, \& D. Milne (Kluwer, Dordrecht), 249

Coulson, I. M., \& Caldwell, J. A. R. 1985a, SAAO Circulars, 9, 5

Coulson, I. M., \& Caldwell, J. A. R. 1985b, MNRAS, 216, 671

Dean, J. F., Warren, P. R., \& Cousins, A. W. J. 1978, MNRAS, 183, 569

Di Benedetto, G. P. 1997, ApJ, 486, 60

Epchtein, N., Deul, E., Derriere, S., et al. 1999, A\&A, 349, 236

Eggen, O. J. 1983, AJ, 88, 379
Feast, M. W. 1999, PASP, 111, 775

Feast, M. W., \& Walker, A. R. 1987, ARA\&A, 25, 345

Fernie, J. D. 1990, ApJS, 72, 153

Fernie, J. D. 1995, AJ, 110, 2361

Fiorentino, G., Caputo, F., Marconi, M., \& Musella, I. 2002, ApJ, 576, 402

Fouqué, P., \& Gieren, W. P. 1997, A\&A, 320, 799 (FG97)

Fouqué, P., Storm, J., \& Gieren, W. P. 2003, in Stellar Candles, ed. D. Alloin, \& W. Gieren; Lecture Notes in Physics (Springer Verlag), 635,21

Freedman, W. L., \& Madore, B. F. 1990, ApJ, 365, 186

Freedman, W. L., Madore, B. F., Gibson, B. K., et al. 2001, ApJ, 553, 47

Fry, A. M., \& Carney, B. W. 1997, AJ, 113, 1073

Gieren, W. P. 1981a, ApJS, 46, 287

Gieren, W. P. 1981b, ApJS, 47, 315

Gieren, W. P. 1985, ApJ, 295, 507

Gieren, W. P., Fouqué, P., \& Gómez, M. 1997, ApJ, 488, 74 (GFG97)

Gieren, W. P., Fouqué, P., \& Gómez, M. 1998, ApJ, 496, 17

Gieren, W. P., Barnes, T. G., \& Moffett, T. J. 1993, ApJ, 418, 135

Gieren, W. P., Storm, J., Fouqué, P., Mennickent, R., \& Gómez, M. 2000, ApJ, 533, L107

Gould, A. 1994, ApJ, 426, 542

Groenewegen, M. A. T. 1999, A\&AS, 139, 245

Groenewegen, M. A. T. 2000, A\&A, 363, 901

Hindsley, R. B., \& Bell, R. A. 1986, PASP, 98, 881

Hindsley, R. B., \& Bell, R. A. 1989, ApJ, 341, 1004

Isobe, T., Feigelson, E. D., Akritas, M. G., \& Babu, G. J. 1990, ApJ, 364, 104

Jarrett, T. H., Chester, T., Cutri, R., et al. 2000, AJ, 119, 2498

Kennicutt, K. C., Stetson, P. B., Saha, A., et al. 1998, ApJ, 498, 181

Kervella, P., Coudé du Foresto, V., Perrin, G., et al. 2001, A\&A, 367, 876

Kiss, L. L. 1998, MNRAS, 297, 825

Kurtz, M. J., \& Mink, D. J. 1998, PASP, 110, 934

Kurucz, R. L. 1993, ATLAS9 Stellar Atmosphere Programs and $2 \mathrm{~km} \mathrm{~s}^{-1}$ Grid (Cambridge: Smithsonian Astrophys. Obs.) 
Kochanek, C. S. 1997, ApJ, 491, 13

Lane, B. F., Creech-Eakman, M. J., \& Nordgren, T. E. 2002, ApJ, 573, 330

Laney, C. D., \& Stobie, R. S. 1992, A\&AS, 93, 93

Laney, C. D., \& Stobie, R. S. 1993, MNRAS, 263, 921

Laney, C. D., \& Stobie, R. S. 1994, MNRAS, 266, 441

Laney, C. D., \& Stobie, R. S. 1995, MNRAS, 274, 337

Larsen, S. S., Clausen, J. V., \& Storm, J. 2000, A\&A, 364, 455

Latham, D. W. 1985, in Stellar Radial Velocities, ed. A. G. D. Philip, \& D. W. Latham (Schenectady: L. Davis Press), IAU Coll., 88, 21

Latham, D. W. 1992, in Complementary Approaches to Binary and Multiple Star Research, ed. H. McAlister, \& W. Hartkopf (San Francisco: ASP), ASP Conf. Ser., 32, 110

Latham, D. W., Stefanik, R. P., Torres, et al. 2002, AJ, 124, 1144

Lejeuene, Th., Cuisinier, F., \& Buser, R. 1998, A\&AS, 130, 65

Lloyd Evans, T. 1980, SAAO Circulars, 1, 257

Luck, R. E., Moffett, T. J., Barnes, T. G., \& Gieren, W. P. 1998, AJ, 115,605

Luck, R. E., Gieren, W. P., Andrievky, S. M., et al. 2003, A\&A, 401, 939

Madore, B. F. 1975, ApJS, 29, 219

Metzger, M. R., Caldwell, J. A. R., McCarthy, J. K., \& Schecter, P. L. 1991, ApJS, 76, 803

Metzger, M. R., Caldwell, J. A. R., \& Schecter, P. L. 1992, AJ, 103, 529

Moffett, T. J., \& Barnes, T. G. 1984, ApJS, 55, 389

Nordgren, T. E., Lane, B. F., Hindsley, R. B., \& Kervella, P. 2002, ApJ, 123, 3380
Nordström, B., Latham, D. W., Morse, J. A., et al. 1994, A\&A, 287, 338

Parsons, S. B. 1972, ApJ, 174, 57

Pel, J. W. 1976, A\&AS, 24, 413

Pont, F., Mayor, M., \& Burki, G. 1994, A\&A, 285, 415

Saha, A., Sandage, A., Tammann, G. A., et al. 2001, ApJ, 562, 314

Saio, H., \& Gautschy, A. 1998, ApJ, 498, 360

Sandage, A., Bell, R. A., \& Tripicco, M. J. 1999, ApJ, 522, 250

Sabbey, C. N., Sasselov, D. D., Fieldus, M. S., et al. 1995, ApJ, 446, 250

Sasselov, D. D., Beaulieu, J. P., Renault, C., et al. 1997, A\&A, 324, 471

Schechter, P. L., Avruch, I. M., Caldwell, J. A. R., \& Keane, M. J. 1992, AJ, 104, 1930

Schlegel, D. J., Finkbeiner, D. P., \& Davis, M. 1998, ApJ, 500, 525

Storm, J., Carney, B. W., Gieren, W. P., et al. 2004, A\&A, 415, 521

Tammann, G. A., Sandage, A., \& Reindl, B. 2003, A\&A, 404, 423

Turner, D. G., \& Burke, J. F. 2002, AJ, 124, 2931

Udalski, A., Soszyński, I., Szymański, M., et al. 1999a, Acta Astron., 49, 223

Udalski, A., Soszyński, I., Szymański, M., et al. 1999b, Acta Astron., 49,437

Udalski, A., Wyrzykowski, L. M., Pietrzyński, et al. 2001, Acta Astron., 51, 221

Welch, D. L., Wieland, F., McAlary, C. W., et al. 1984, ApJS, 54, 547

Welch, D. L. 1985, Ph.D. Thesis, University of Toronto

Welch, D. L. 1994, AJ, 108, 1421

Zaritsky, D., Harris, J., Thompson, I. B., Grebel, E. K., \& Massey, P. 2002, AJ, 123, 855 